\title{
SOX4 and SMARCA4 cooperatively regulate PI3k signaling through transcriptional activation of TGFBR2
}

\author{
Gaurav A. Mehta ${ }^{1,2}$, Steven P. Angus $\mathbb{D}^{3}{ }^{3}$, Christen A. Khella $\mathbb{D}^{1,2}$, Kevin Tong ${ }^{2,4}$, Pooja Khanna ${ }^{1,2}$, Shelley A. H. Dixon ${ }^{3}$, Michael P. Verzi ${ }^{2,4}$,
} Gary L. Johnson iD $^{5}$ and Michael L. Gatza $\mathbb{i D}^{1,2 \bowtie}$

Dysregulation of PI3K/Akt signaling is a dominant feature in basal-like or triple-negative breast cancers (TNBC). However, the mechanisms regulating this pathway are largely unknown in this subset of aggressive tumors. Here we demonstrate that the transcription factor SOX4 is a key regulator of PI3K signaling in TNBC. Genomic and proteomic analyses coupled with mechanistic studies identified TGFBR2 as a direct transcriptional target of SOX4 and demonstrated that TGFBR2 is required to mediate SOX4dependent PI3K signaling. We further report that SOX4 and the SWI/SNF ATPase SMARCA4, which are uniformly overexpressed in basal-like tumors, form a previously unreported complex that is required to maintain an open chromatin conformation at the TGFBR2 regulatory regions in order to mediate TGFBR2 expression and PI3K signaling. Collectively, our findings delineate the mechanism by which SOX4 and SMARCA4 cooperatively regulate PI3K/Akt signaling and suggest that this complex may play an essential role in TNBC genesis and/or progression.

npj Breast Cancer (2021)7:40; https://doi.org/10.1038/s41523-021-00248-2

\section{INTRODUCTION}

Triple-negative breast cancer (TNBC), which is largely synonymous with the basal-like molecular subtype of breast cancer, accounts for $10-15 \%$ of all breast-cancer cases ${ }^{1}$. These aggressive tumors are predominant in younger women and women of African American descent and are characterized by poor clinical outcome, accounting for $\sim 1$-in-4 breast-cancer-related deaths ${ }^{2,3}$. Previous studies, including those from The Cancer Genome Atlas (TCGA) project and our own work, have reported increased and uniform activation of phosphatidylinositol-3-OH kinase (PI3K) signaling in basal-like breast tumors ${ }^{3-6}$. This pathway mediates multiple oncogenic processes including proliferation, metabolism, motility, and genome instability ${ }^{3,7,8}$. While PIK3CA, which encodes for the oncogenic p110a catalytic subunit of the kinase, is the most commonly mutated gene in breast cancer ${ }^{3}$, activating mutations occur at a low incidence ( 9\%) in basal-like tumors suggesting that other mechanisms contribute to altered signaling in these tumors $^{3,9}$. Consistent with this argument, multi-platform genomic analyses have identified copy-number alterations or mutations in repressors of PI3K signaling including PTEN (35\%) as well as mutations in known drivers of the pathway including EGFR (7\%), ERBB2 (4\%), IGFR1 (2\%), and others in basal-like tumors ${ }^{3,6}$. Despite these observations, predominant mechanisms regulating activation of this pathway have not been identified in TNBC or basal-like tumors. While inhibition of PI3K/Akt/MTOR signaling has been shown to be effective in preclinical studies and in ER+ breast cancers, similar clinical success has not been achieved for $\mathrm{TNBC}^{7,10-15}$. These clinical results suggest that understanding and targeting these additional mechanisms of PI3K pathway regulation and/or complementary pathways will be important for optimizing therapeutic strategies for TNBC patients.

SOX4 is a well-established oncogene and a member of the SOX C family of SRY-related HMG-box (SOX) transcription factors ${ }^{16}$.
Increased expression of SOX4 has been shown to be associated with malignant transformation and metastasis in several cancer types including breast ${ }^{5,17-20}$, prostate ${ }^{21,22}$, acute lymphoblastic leukemia ${ }^{23}$, and melanoma ${ }^{24}$. In the context of breast cancer, overexpression of SOX4 corresponds with poor overall survival, particularly in basal-like or TNBC tumors ${ }^{18,20,25}$. The pro-oncogenic function of SOX4 in breast cancer is widely attributed to its ability to modulate epithelial-to-mesenchymal transition (EMT), activation of multiple pro-proliferative or pro-survival signaling pathways, increased angiogenesis as well as its role in regulating cancer cell stemness ${ }^{16-20}$. Consistent with the observed effect of SOX4 on multiple oncogenic signaling pathways, we recently showed that SOX4 is an essential regulator of PI3K/Akt signaling in basal-like tumors ${ }^{5}$. These previous studies demonstrated that SOX4 expression is increased in basal-like breast tumors with high PI3K activity, independent of genomic alteration in commonly altered PI3K/Akt regulatory genes including PIK3CA and PTEN, and that siRNA-mediated silencing of SOX4 abrogates the activation of this pathway in basal-like cell lines; however, the mechanism(s) by which SOX4 regulates this pathway in TNBC remains unknown.

SWI/SNF chromatin remodeling enzymes are multi-subunit and evolutionarily conserved complexes that remodel chromatin by utilizing energy derived from ATP hydrolysis and render sites accessible to transcription factor binding in order to promote gene expression ${ }^{26,27}$. Mutations in subunits of the SWI/SNF chromatin remodeling complexes have been reported in nearly $20 \%$ of all human cancers, highlighting their important roles in tumorigenesis $^{28-30}$. Consistent with these general observations, recent studies have demonstrated that increased expression of SMARCA4, which encodes the catalytic ATPase subunit of the SWI/ SNF complex, is associated with poor prognosis in breast cancer ${ }^{31-33}$. Mechanistically, SMARCA4 has been reported to play an oncogenic role in breast cancer by regulating critical aspects of breast-

\footnotetext{
${ }^{1}$ Department of Radiation Oncology, Robert Wood Johnson Medical School, New Brunswick, NJ, USA. ${ }^{2}$ Rutgers Cancer Institute of New Jersey, New Brunswick, NJ, USA. ${ }^{3}$ Department of Pediatrics, Indiana University School of Medicine, Indianapolis, IN, USA. ${ }^{4}$ Department of Genetics, Human Genetics Institute of New Jersey, Rutgers, The State University of New Jersey, Piscataway, NJ, USA. ${ }^{5}$ Department of Pharmacology, University of North Carolina School of Medicine, Chapel Hill, NC, USA. ${ }^{\circledR}$ email: Michael.gatza@cinj. rutgers.edu
} 
cancer biology including lipid metabolism, proliferation, and resistance to chemotherapeutic drugs ${ }^{34,35}$. Although SMARCA4 has been shown to regulate several oncogenic properties in breast cancer, the mechanisms by which it promotes these processes in mammary tumorigenesis, including potential effects on PI3K/Akt signaling, remains unknown.

The goal of the current study was to define the mechanism and co-factors required for SOX4-mediated activation of PI3K/Akt signaling in TNBC. To achieve this goal, mRNA sequencing (RNAseq) and kinome profiling coupled with molecular analyses identified and determined that SOX4-mediated activation of PI3K/ Akt signaling is dependent on transcriptional regulation of transforming growth factor $\beta$ receptor 2 (TGFBR2) by SOX4. At the molecular level, our data demonstrate that SOX4 recruits SMARCA4 to the TGFBR2 promoter and enhancer in order to mediate chromatin remodeling and activation of TGFBR2 expression. Given that our data indicate that SOX4 and SMARCA4 are uniformly activated in basal-like breast tumors, our findings not only delineate a mechanism by which SOX4 and SMARCA4 mediate PI3K activity in TNBC or basal-like breast cancers but have also identified a novel complex by which SOX4 cooperates with SMARCA4 to modulate transcriptional activation and oncogenic signaling in basal-like breast cancer.

\section{RESULTS}

SOX4 expression is upregulated in basal-like tumors and associated with increased PI3K signaling

Previous studies have demonstrated that basal-like tumors are characterized by high PI3K/Akt signaling ${ }^{3-5}$. More recently, we reported that SOX4 can mediate PI3K and Akt signaling in TNBC cell lines ${ }^{5}$. In order to demonstrate the relationship between SOX4 expression and aberrant PI3K/Akt signaling, as well as the association between SOX4 expression and molecular subtype, we examined these relationships in human breast tumors. Patient tumor samples from TCGA $(n=1031)^{3}$ and Molecular Taxonomy of Breast Cancer International Consortium (METABRIC, $n=1992)^{36}$ cohorts were scored for PAM50 subtype ${ }^{37}$, and PI3K pathway activity was calculated using a previously published PI3K gene

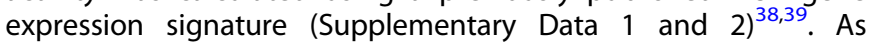
illustrated in Fig. 1, when tumor samples were dichotomized into SOX4 high (top quartile) and low (bottom quartile) expressing subgroups, PI3K signaling was significantly upregulated in both the TCGA (Fig. $1 \mathrm{a} ; p=3.0 \times 10^{-27}$ ) and METABRIC (Fig. 1b; $p=$ $4.7 \times 10^{-70}$ ) cohorts in SOX4 high expressing tumors. As expected $^{5}$, increased SOX4 expression was predominantly observed in basal-like tumors (Fig. 1a, b) and analysis of the TCGA dataset demonstrated that more than $88.1 \%$ of basal-like tumors $(n=179)$ were characterized by increased $\left(p=6.35 \times \mathrm{e}^{-20}\right)$ SOX4 expression relative to the median expression in adjacent normal breast tissue $(n=94)$ (Fig. 1c and Supplementary Data 1).

In order to identify cell lines for in vitro studies, we used data from publicly available gene expression and proteome profiling studies to examine SOX4 mRNA (Supplementary Fig. 1a) and protein (Supplementary Fig. 1b) expression in a panel of breastcancer cell lines. Western blot analyses were performed to validate SOX4 expression in a subset of basal-like cell lines that demonstrated relatively high (HCC1143, HCC1954, MDAMB468, HCC1395, HCC38, HCC70) or low (BT20, MDAMB231) SOX4 expression (Supplementary Fig. 1C). Based on these analyses, as well as previously published PI3K activity scores, HCC1143 and HCC1954 were identified as basal-like breast-cancer cell lines with high SOX4 expression and high PI3K activity and selected for in vitro studies ${ }^{5,40}$. As expected RNAi-mediated silencing reduced SOX4 mRNA expression by $67.3 \%$ in $\operatorname{HCC} 1143(p=0.0014)$ and $72.3 \%$ in HCC1954 $(p=0.0051)$ cells. This corresponded with a significant $34.6 \%(p=0.0019)$ and $40.1 \%(p=0.0067)$ reduction in protein expression in each cell line (Fig. 1e, $f$ and Supplementary Fig. 2a, b). Consistent with predictions from in silico analyses of human tumors, siRNA-mediated silencing of SOX4 in HCC1143 $(p=0.0019)$ cells significantly reduced Akt phosphorylation at Ser473 $(p=0.0002)$ and decreased phosphorylation of downstream PI3K pathway proteins including pP70S6K at Thr389 ( $p=$ $0.0006)$ and p4EBP1 at Thr37/46 ( $p=0.0043)$; siRNA-mediated silencing of SOX4 did not have an effect on total Akt, P70S6K, or 4EBP1 levels (Fig. 1e and Supplementary Fig. 2a). Likewise, siRNAmediated silencing of SOX4 in HCC1954 cells $(p=0.0067)$ resulted in a similar reduction in Akt signaling as evident by decreased phosphorylation of pAkt (Ser473; $p=0.0181$ ), pP70S6K (Thr389; $p=0.0222$ ), and p4EBP1 (Thr37/46; $p=0.0108$ ) with no concomitant change in total Akt, P70S6K, or 4EBP1 expression (Fig. 1e and Supplementary Fig. 2b). In order to validate the specificity of these findings, we engineered HCC1143 and HCC1954 cells to express one of two unique tetracycline-inducible shRNA against SOX4 (referred to as sh-1 and sh-2); as expected, parental and tetinducible shRNA expressing HCC1143 and HCC1954 cells show similar basal levels of SOX4, pAkt, and total Akt protein expression (Supplementary Fig. 2c, d). As illustrated in Supplementary Fig. 2e, doxycycline (dox) treatment resulted in significant reduction of SOX4 mRNA levels in sh-1 (83.0\% reduction, $p=0.0049)$ or sh-2 (65.01\% reduction, $p=0.0099$ ) expressing HCC1143 cells and sh-1 (86.45\% reduction, $p=0.0008)$ or sh $-2(73.0 \%$ reduction, $p=$ 0.0013 ) expressing HCC1954 cell lines (Supplementary Fig. 2e). In agreement with siRNA knockdown, shRNA-mediated depletion of SOX4 resulted in the significant decrease in SOX4 protein expression as well as Akt (S473) phosphorylation in both HCC1143 (Supplementary Fig. 2f) and HCC1954 (Supplementary Fig. $2 g$ ) cell lines.

\section{Identification of the SOX4-activated kinome}

Given that SOX4 is a transcription factor, we hypothesized that SOX4 mediates its effects on PI3K/Akt signaling by regulating expression of upstream kinases. In order to identify kinases regulated by SOX4 that contribute to increased $\mathrm{PI} 3 \mathrm{~K} / \mathrm{Akt}$ signaling, we performed kinome profiling using multiplexed inhibitor beads coupled with mass spectrometry (MIB/MS). MIB/MS is a chemicalproteomic-based approach, which utilizes multiple kinase inhibitors to enrich the functional kinome from tumor or cell lysates by affinity chromatography followed by quantitative mass spectrometry $(\mathrm{MS})^{41}$. Using this approach, we identified 208 enriched kinases in siSOX4 or siControl-treated HCC1143 cells. Of these, the MIB binding of 30 kinases was significantly $(p<0.05)$ altered following siRNA-mediated silencing of SOX4 relative to siControltreated cells. Eighteen kinases, including BMPR1A ${ }^{42}, \mathrm{LCK}^{43}$, $\mathrm{ACVR}_{1}{ }^{44}$, and EGFR ${ }^{45}$, which are known regulators 'of PI3K' signaling, were significantly depleted while twelve kinases including several belonging to MAPK family showed increased enrichment following siSOX4 treatment (Fig. 2a and Supplementary Data 3).

To determine if any of the depleted kinases from MIB/MS analysis are transcriptional targets of SOX4, and thus directly regulated by SOX4 activity, we performed transcriptome profiling by RNA sequencing in HCC1143 cells treated with siRNA against SOX4 or a control siRNA. Differential gene expression analyses of RNAseq data identified TGFBR2 $(p=0.009)$ as the only gene among the MIB/MS hits that was significantly downregulated following siRNA-mediated silencing of SOX4 (Fig. $2 \mathrm{~b}$ and Supplementary Data 4).

In order to validate these omics-based analyses, the effect of siRNA-mediated knockdown of SOX4 on TGFBR2 expression was examined in HCC1143 and HCC1954 cell lines. Quantitative realtime PCR (qRT-PCR) analysis confirmed downregulation of TGFBR2 expression at the transcript level in both HCC1143 $(62.0 \%$ reduction, $p=0.014$ ) (Fig. $2 \mathrm{c}$ ) and $\mathrm{HCC} 1954$ (74.6\% reduction, 
a.

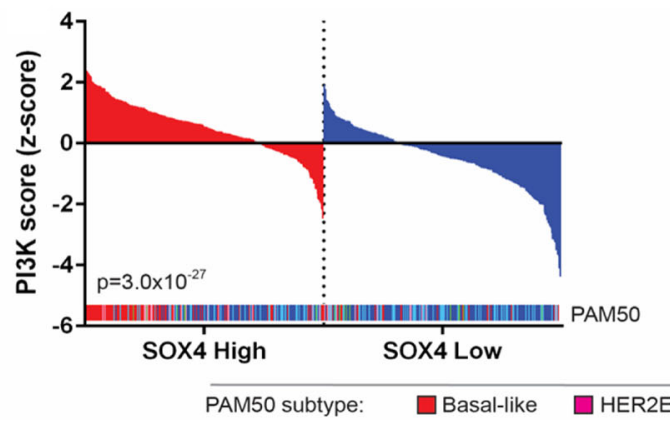

c.

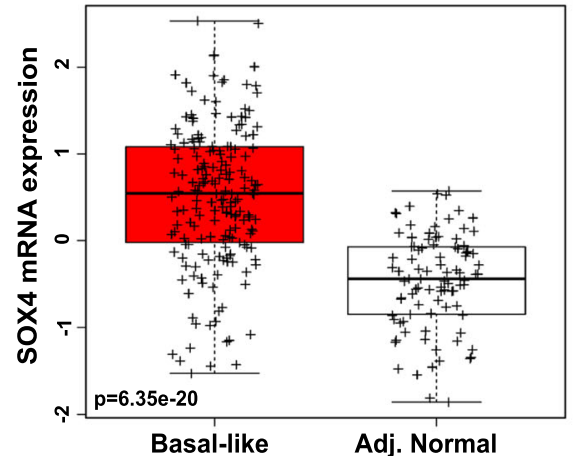

METABRIC

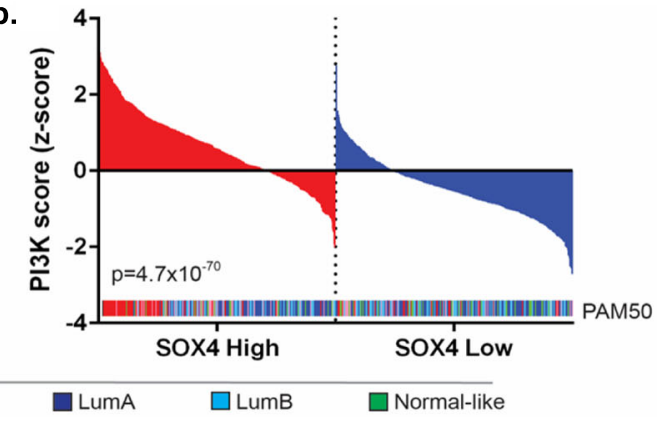

d.

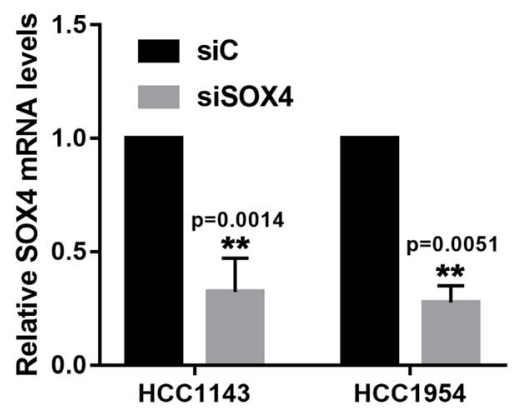

e.

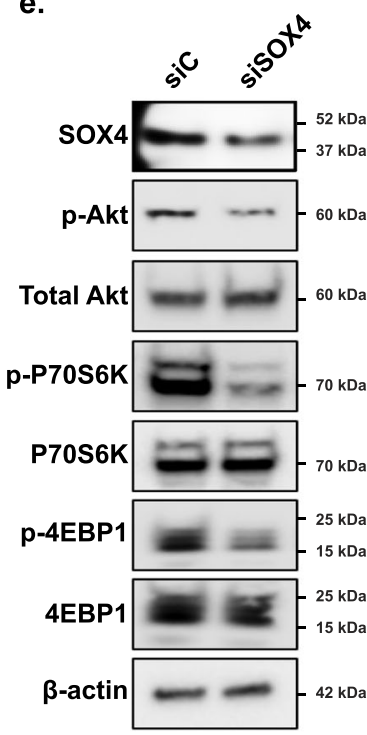

HCC1143 f.

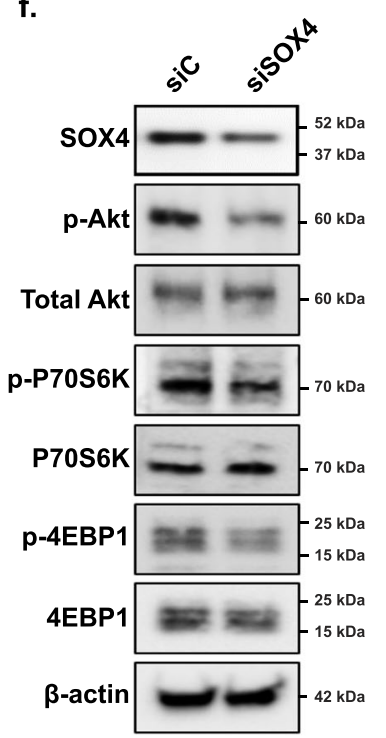

HCC1954

Fig. 1 SOX4 regulates the PI3K/Akt pathway activity in basal-like breast cancer. PI3K pathway activity is significantly upregulated in tumor samples with high SOX4 expression (top quartile) compared to samples with low (bottom quartile) SOX4 expression in the a TCGA ( $n=1031$, $\left.p=3.0 \times 10^{-27}\right)$ and $\mathbf{b}$ METABRIC $\left(n=1991, p=4.7 \times 10^{-70}\right)$ datasets. PAM50 subtype is shown in the inset of $\mathbf{a}$ and $\mathbf{b}$ and demonstrates that samples with high SOX4 expression are predominantly basal-like tumors (red) in both datasets. c SOX4 expression is significantly upregulated in $88.1 \%$ of in basal-like breast tumors $(n=179)$ compared to the median expression in adjacent normal tissue $(n=94)$ from the TCGA dataset $\left(p=6.35 \times 10^{-20}\right)$. d siRNA-mediated silencing of SOX4 significantly reduced SOX4 expression in HCC1143 $(p=0.001)$ and HCC1954 $(p=0.005)$ TNBC cell lines. Representative western blot analysis demonstrating significant reduction in SOX4, pAkt, pS6K, and p4EBP1 expression in both e HCC1143 and f HCC1954 cell lines.

$p=0.011$ ) (Fig. 2d); a similar percent reduction was also observed in these cells at the protein level in HCC1143 (Fig. 2e and Supplementary Fig. 3a; $p=0.005$ ) and HCC1954 (Fig. 2f and Supplementary Fig. 3b; $p=0.007$ ) cells. Importantly a significant reduction in p-SMAD2 was observed in both HCC1143 (Fig. 2e and
Supplementary Fig. 3a; $p=0.01$ ) and HCC1954 (Fig. 2f and Supplementary Fig. 3b; $p=0.01$ ) cells indicating that TGF $\beta$ signaling is functionally inhibited by SOX4 repression. Consistent with these results, shRNA-mediated silencing of SOX4, using two independent tet-inducible shRNA, also resulted in a significant 


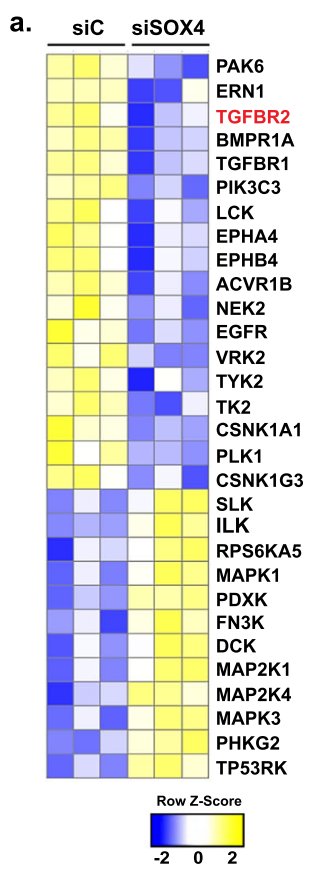

c.

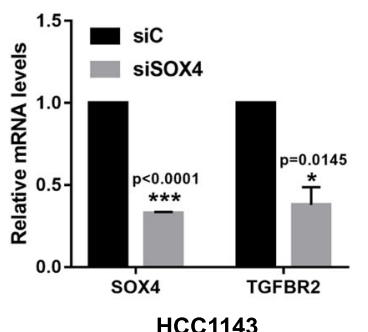

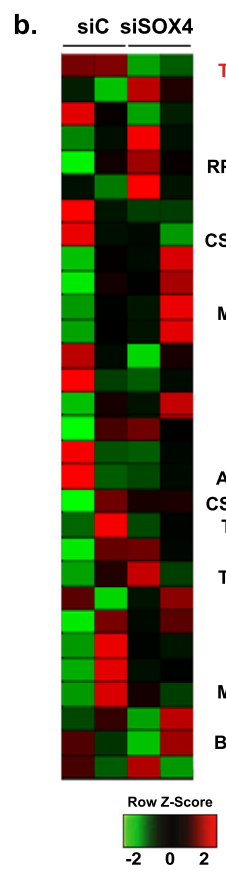

d.

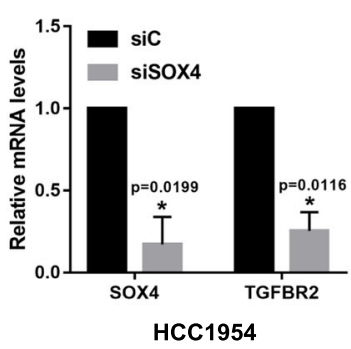

e.

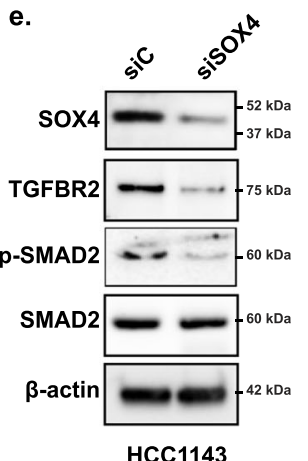

HCC1143

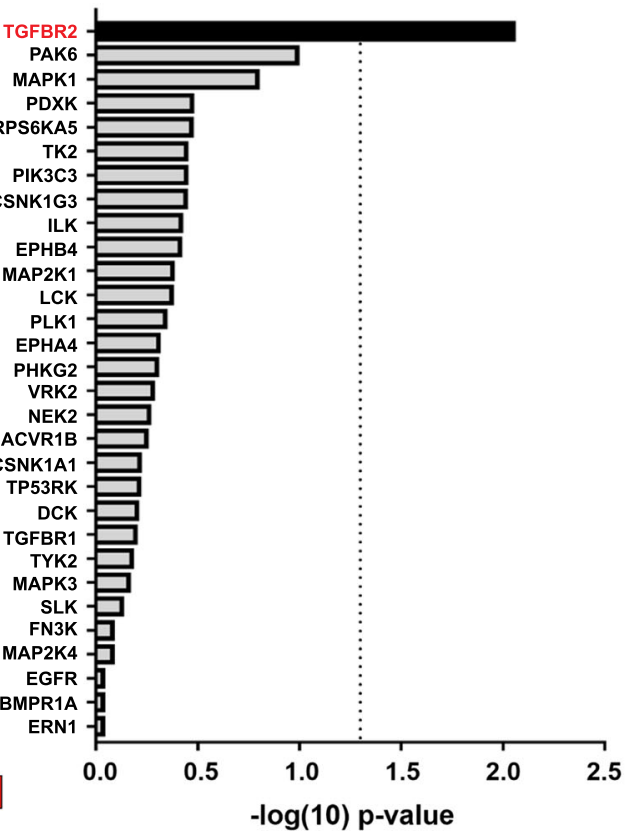

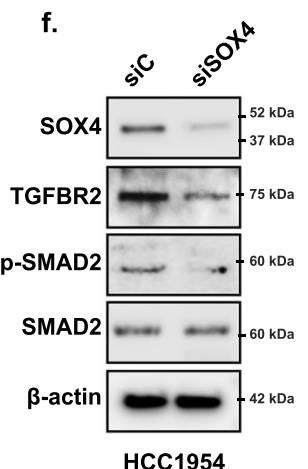

Fig. 2 TGFBR2 is a downstream target gene of SOX4. a Profiling of the drug-able kinome by MIB/MS analysis in HCC1143 cells identified 18 kinases with significantly reduced expression and/or affinity for Type I kinase inhibitor bait $(p<0.05)$ and 12 kinases with increased expression/ affinity following siRNA-mediated silencing of SOX4 $(n=3,96 \mathrm{~h})$ relative to siControl-treated samples $(n=3)$; kinase enrichment score is shown in yellow and kinase depletion score in blue. $\mathbf{b}$ Differential gene expression analyses of RNAseq data for the significantly altered kinases from MIB/MS analysis from siSOX4- and siControl-treated HCC1143 cells (96h); high mRNA expression is shown in red and low expression in green. $\mathbf{c}$ HCC1143 and d HCC1954 cells demonstrate significant reduction (unpaired t-test) in SOX4 ( $p<0.0001 ; p=0.019)$ and TGFBR2 ( $p=0.014 ; p=0.012$ ) expression in siSOX4-treated cells compared to siControl-treated cell by qRT-PCR; data are presented as mean \pm SD and normalized for GAPDH $(n=3)$. e HCC1143 and f HCC1954 cells showing reduced SOX4 and TGFBR2 protein expression by western blot analyses in siSOX4-treated cells relative to siControl-treated cells.

downregulation of TGFBR2 protein expression in both cell lines (Supplementary Fig. 3c and Supplementary Fig. 3d).

\section{SOX4-induced TGFBR2 expression promotes PI3K/Akt signaling}

To investigate the impact of TGFBR2 on SOX4 activation of the $\mathrm{PI} 3 \mathrm{~K} /$ Akt pathway in TNBC, we first inhibited TGFBR2 expression by siRNA independent of SOX4 perturbation. RNAi-mediated suppression of TGFBR2 significantly reduced PI3K signaling as apparent by decreased phosphorylation of downstream markers of the PI3K pathway including pAkt (Ser473) (HCC1143, $p=0.018$; HCC1954, $p=0.007)$, pP70S6K (Thr389) (HCC1143, $p=0.023$; HCC1954, $p=0.012$ ), and p4EBP1 (Thr37/46) (HCC1143, $p=$ 0.035; HCC1954, $p=0.025$ ) in HCC1143 (Fig. 3a and Supplementary Fig. 4a) and HCC1954 (Fig. 3b and Supplementary Fig. 4b) cells. As shown in Fig. 3, total protein levels for Akt, P70S6K, and 4EBP1 remain unchanged and this effect was consistent in both cell lines (Fig. 3a, b).
In order to test the hypothesis that SOX4-mediated activation of PI3K signaling is dependent on TGFBR2 expression, SOX4 was depleted in HCC1143 or HCC1954 cell lines by siRNA while TGFBR2 was concurrently overexpressed by lentiviral transduction. As expected, SOX4 knockdown by RNAi (siSOX4) significantly reduced the expression of TGFBR2 and phosphorylation of Akt (Ser473) in both HCC1143 (Fig. 3c) and HCC1954 (Fig. 3d) cell lines. Importantly, we determined that TGFBR2 overexpression in conjunction with SOX4 knockdown was able to rescue PI3K/Akt signaling, as illustrated by restored pAkt (Ser473) levels, in each cell line (Fig. 3c, d).

SOX4 regulates TGFBR2 expression by binding to the HMGbox domain in its promoter and enhancer regions

Given the dependency of SOX4 on TGFBR2 as a critical regulator of PI3K signaling, we next investigated the mechanism by which SOX4 regulates TGFBR2 expression. The SOX4 protein contains an HMG-box domain that enables it to interact with the A/TA/TCAAA/ 

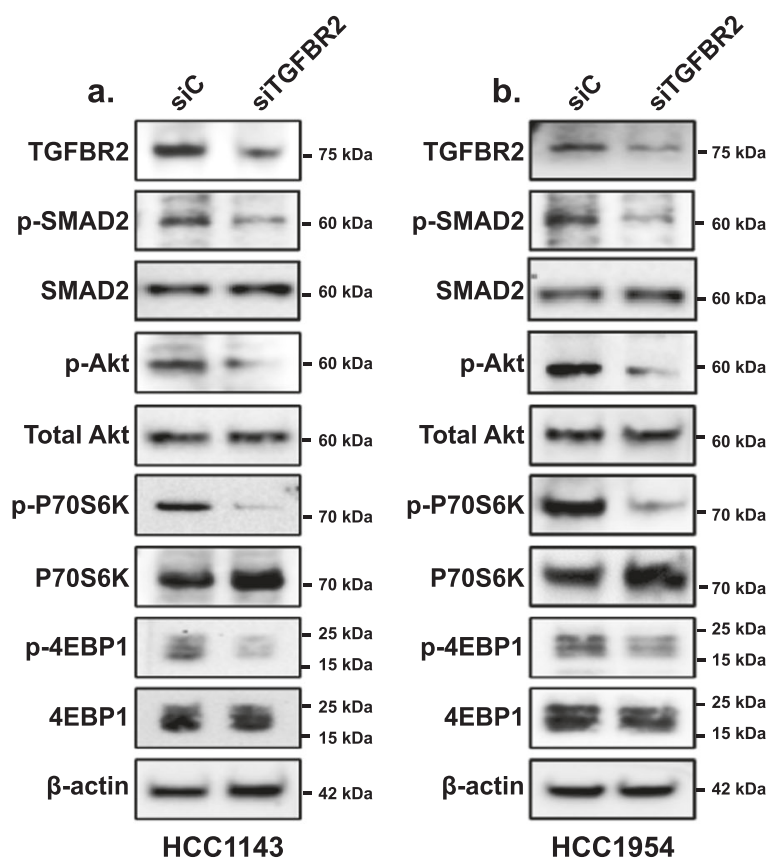

c.

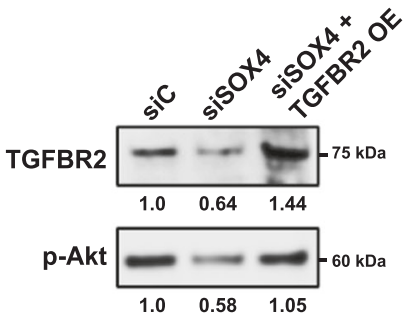

Total Akt $--60 \mathrm{kDa}$

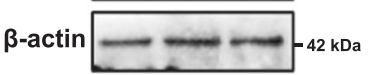

HCC1143 d.
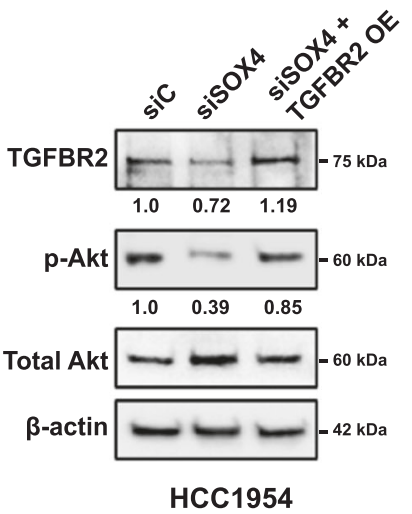

Fig. 3 SOX4 mediates activation of the PI3K pathway through TGFBR2. a Western blot analysis showing downregulation of the PI3K pathway activity as indicated by decreased expression of PI3K pathway downstream components in HCC1143 following siTGFBR2 treatment $(96 \mathrm{~h})$ relative to siControl treatment. p-SMAD2 downregulation is used as a positive control for TGFBR2 protein depletion. b Similar results were observed in HCC1954 cells. c Lentiviral overexpression of TGFBR2 restores pAkt levels in siSOX4 (96h) treated HCC1143 cells. d Consistent results were observed in HCC1954 cells.

TG motif in the minor groove of the DNA to mediate transcription of its target genes ${ }^{16,22}$. Therefore, we first screened the genome upstream of the TGFBR2 transcriptional start site for potential functional genetic elements harboring the SOX4 binding motif. These analyses identified putative SOX4 binding motifs (AACAAAG) in the distal promoter $(-2265 \mathrm{bp}$ site) and enhancer regions ( -8254 bp site) (Fig. 4a), suggesting that SOX4 may potentially bind and regulate TGFBR2 expression through these conserved motif sequences.

Based on the identification of the putative binding sites, we next examined the capacity of SOX4 to bind to the TGFBR2 promoter and enhancer by performing chromatin immunoprecipitation followed by quantitative-PCR (ChIP-qPCR) analysis. To circumvent the issue of antibody non-specificity and crossreactivity during ChIP analysis, CRISPR/cas9 genome editing was used to add a V5 epitope tag to the carboxyl (C)-terminus of endogenous SOX4 in HCC1143 cells (hereafter referred to as HCC $1143^{\text {SOX4-V5 }}$ cell line) (Fig. 4b and Supplementary Fig. 5a). DNA sequencing (Supplementary Fig. 5a), western blot analyses (Fig. 4c), and immunofluorescence microscopy (Fig. 4d) confirmed expression of the SOX4-V5 protein in HCC $1143^{\text {SOX4-V5 }}$ cells but not in the parental HCC1143 cell line. Finally, to ensure that the C-terminal epitope tag did not disrupt the observed effect of SOX4 on PI3K/Akt signaling and/or TGFBR2 expression, we examined the effect of siRNA-mediated silencing of SOX4 on these processes. SOX4 mRNA (Fig. 4e; $p<0.0001$ ) and protein levels (Fig. $4 \mathrm{f}$ and Supplementary Fig. 5b, $p=0.002$ ) were significantly depleted upon siRNA-mediated silencing of SOX4 and, as expected, V5 protein expression (Fig. $4 \mathrm{f}$ and Supplementary Fig. $5 b, p=0.001$ ) was significantly reduced in $\mathrm{HCC} 1143^{\text {SOX4-V5 }}$ cells following siSOX4 treatment when compared to siControl-treated cells. Likewise, we observed a significant reduction in TGFBR2 protein expression $(p=0.031)$, which we have established as a downstream target of SOX4, as well as phosphorylated Akt (S473) $(p=0.01)$, P70S6K (Thr389) $(p=0.0007)$, and 4EBP1 (Thr37/46) $(p=0.01)$ proteins upon SOX4 inhibition (Fig. $4 \mathrm{f}$ and Supplementary Fig. 5b). These data indicate that addition of the V 5 epitope tag to the C-terminal domain of endogenous SOX4 does not interfere with these specific functions of the SOX4 protein in $\mathrm{HCC} 1143^{\text {SOX4 } 455}$ cells, and suggest that the V5 epitope could be used as a marker to study SOX4 function in this cell line.

Based on these results, we next used the HCC1143 $3^{\text {sox4-v5 }}$ cell line to examine the relative enrichment of SOX4-V5 on the TGFBR2 promoter and enhancer. Chromatin Immunoprecipitation (ChIP) using an antibody against the V5 epitope was used to isolate V5tagged SOX4. Quantitative RT-PCR primers spanning the SOX4 binding motif in the distal promoter and enhancer regions were then used to quantify levels of SOX4 at each site (Fig. 4a). ChIPqPCR analysis revealed a greater than threefold significant enrichment $(p=0.008)$ of SOX4 at both the TGFBR2 promoter (Fig. $4 \mathrm{~g})$ and enhancer $(p=0.0098)$ regions relative to the $\lg \mathrm{G}$ control (Fig. 4h).

\section{SMARCA4 and SOX4 form an essential regulatory complex at the TGFBR2 promoter and enhancer}

Regulation of gene expression is dependent on the ability of the cell to modulate the accessibility of the tightly condensed chromatin to sequence-specific transcription factors and basal transcription machinery ${ }^{46,47}$. Since SOX4 lacks the ability to remodel chromatin, we sought to identify its interaction with the cell-specific chromatin remodeling machinery and demonstrate the role of these co-factors in regulating expression of SOX4 target genes, specifically TGFBR2. To identify the SOX4 interactome, we immunoprecipitated SOX4 protein complexes from HCC1143 ${ }^{\text {SOX4-V5 }}$ cells using antibodies against the V5 epitope (or lgG control) followed by LC-MS/MS analysis. Our analyses identified 1700 proteins enriched in the V5 and IgG antibody pull-downs with a peptide FDR of $<1 \%$ (Supplementary Data 5). Interestingly, these analyses identified several components of the SWI/SNF chromatin remodeling complex, including SMARCA4, SMARCB1, SMARCC1, SMARCC2, SMARCD2, SMARCE1, and ACTL6A as potential SOX4 co-factors (Fig. 5a). Given the recognized function of SWI/SNF complex as epigenetic regulatory proteins, 


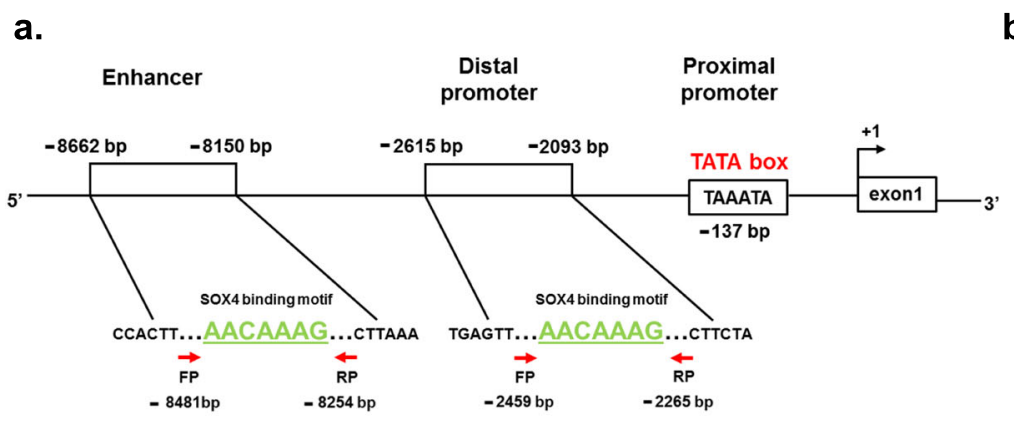

b. 5'...TCACTTTTTTTTTCTCCTCTCCTACCCCCCCCGGCC CTTCTCCCTGCC TGCGCGCCC TTCACGTAGAATCGAG ACCGAGGAGAGGGTTAGGGATAGGCTTACCGTAGGT GAAAACGAGGTTGGAGATGC TGGACTCGAGCCAGTCT CCCGAGATCATCTCGCTCAC... $3^{\prime}$

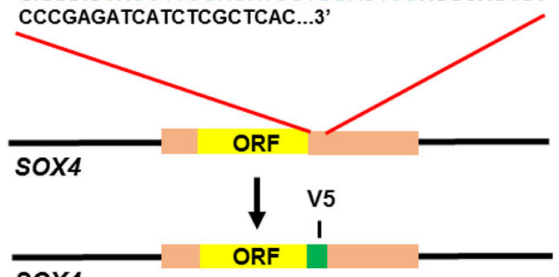

sox4

V5 tag |altered PAM motif | gRNA sequence | Stop codon

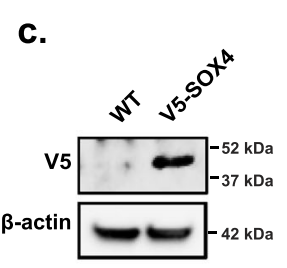

d.
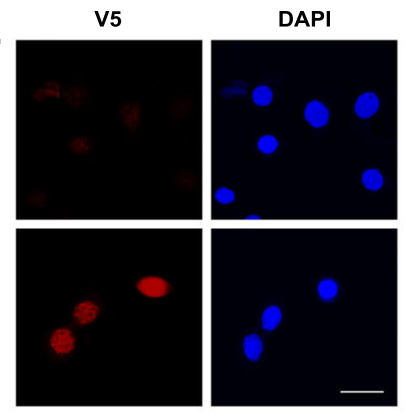

WT

v5-sox4

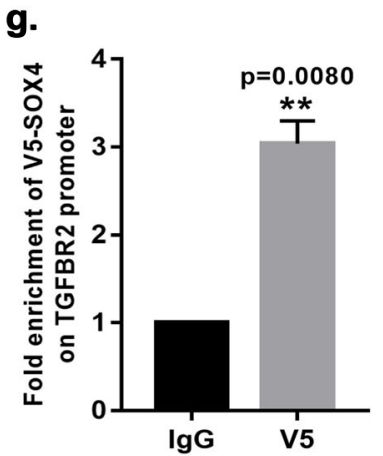

h.

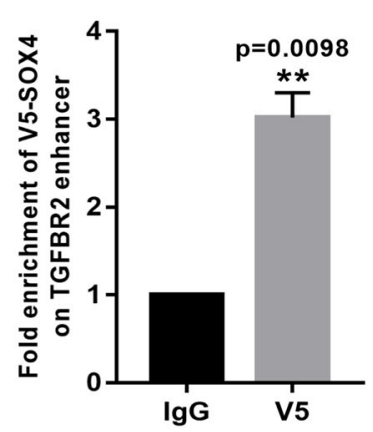

e.

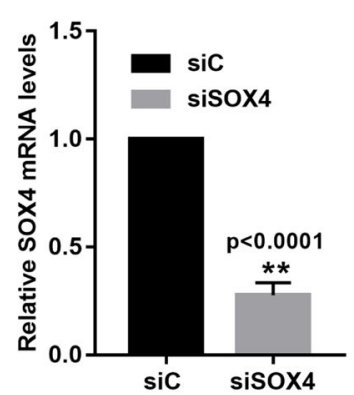

f.

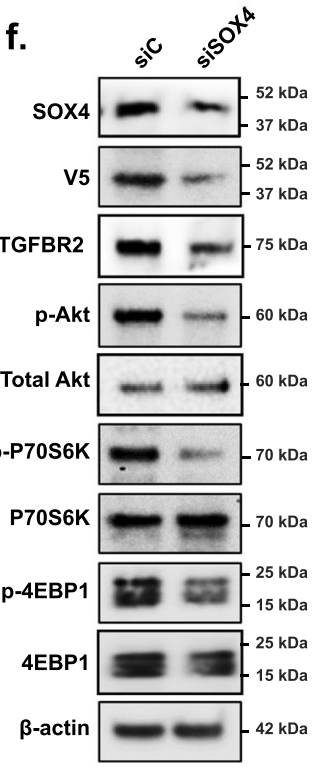

Fig. 4 SOX4 binds to regulatory sequences in TGFBR2 promoter and enhancer region. a Schematic representation of the TGFBR2 regulatory regions upstream from the transcription start site. The proximal promoter with TATA box (red), the SOX4 binding motif, (AACAAAG) in the distal promoter and enhancer (green), and forward/reverse primer locations (red arrows) are noted. b Schematic of the CRISPR/Cas9 genome editing strategy used to insert a C-terminal V5 epitope tag (green) at the $3^{\prime}$ locus immediately upstream of the stop codon (red); the gRNA sequence (blue) and the altered PAM motif (brown) are highlighted. $\mathbf{c}$ Western blot and $\mathbf{d}$ immunofluorescence demonstrate V5 expression in the HCC1143 ${ }^{\text {SOX4-V5 }}$ cell line; no expression is observed in the parental cell. The scale bar is $100 \mu \mathrm{m}$. e qRT-PCR demonstrates that SOX4 mRNA levels are significantly reduced by siSOX4 in HCC1143 ${ }^{\text {SOX4-V5 }}(p<0.0001)$ relative to siControl transfected cells $(50 \mathrm{~nm}, 96 \mathrm{~h})$. f Western blot analyses demonstrated reduced protein expression of SOX4, V5, TGFBR2, and phosphorylated PI3K pathway marker proteins in HCC1143 SOX4-V5 cells transfected with siSOX4 relative to siControl-treated cells $(96 \mathrm{~h})$. g ChIP-qPCR using anti-V5 antibody demonstrated a $3.03-$ fold enrichment ( $p=0.008$, unpaired $t$-test) of V5-tagged Sox4 protein at the TGFBR2 promoter relative to lgG control. $\mathbf{h}$ A 3.01-fold enrichment of V5 protein ( $p=0.01$, unpaired $t$-test) was observed at the TGFBR2 enhancer in HCC1143 ${ }^{\mathrm{SOX} 4-\mathrm{V} 5}$ cells relative to lgG control. Fold enrichment is normalized to PNOC promoter region, which is used as a negative control.

and the noted contribution of several of these proteins to breastcancer pathogenesis ${ }^{48-50}$, we postulate that SOX4 remodels the TGFBR2 regulatory regions by interacting with the functional SWI/ SNF chromatin remodeling complex.

To test this possibility, we first examined the subtype-specific expression of the identified SWI/SNF complex proteins in human breast tumors. Consistent with our previous studies, analyses of gene expression data from the TCGA cohort ( $n=1031$ tumors, $n$ $=94$ adjacent normal tissue) identified significant upregulation of SOX4 expression in basal-like tumors (Fig. 5 b) ${ }^{5}$. More importantly, our analyses identified increased expression of the core components of the SWI/SNF complex SMARCB1, SMARCC1, SMARCD2, and ACTL6A in basal-like tumors compared to other subtypes and adjacent normal tissue (Fig. 5b). These data suggest that the functional SWI/SNF complex may be aberrantly activated in basallike tumors and cooperate with SOX4 to mediate its activity. Interestingly, however, SMARCA4 and SMARCA2, which are the ATPase subunits of this complex, demonstrated an anti-correlated pattern of expression with basal-like tumors expressing high levels of SMARCA4 mRNA but low levels of SMARCA2 transcripts (Fig. 5b). Consistent with these observations, a significant positive correlation was identified between SOX4 and SMARCA4 $(r=0.4, p=8.9 \times$ $\left.10^{-36}\right)$, SMARCB1 $\left(r=0.3, p=1.4 \times 10^{-28}\right)$, SMARCC1 $(r=0.4, p=$ $\left.5.3 \times 10^{-42}\right)$, SMARCD2 $\left(r=0.1, p=1.5 \times 10^{-5}\right)$, and ACTL6A $(r=$ $0.5, p=2.3 \times 10^{-61}$ ) gene expression in basal-like tumors (Fig. $5 b$ ). Conversely, a weak negative correlation was observed between SOX4 and SMARCA2 $\left(r=-0.2, p=3.5 \times 10^{-8}\right)$ mRNA expression in this subset of TCGA tumors (Fig. 5b). Similar gene expression and 
a.

\begin{tabular}{ccc}
\hline $\begin{array}{c}\text { SWI/SNF complex proteins } \\
\text { that interact with SOX4 and } \\
\text { identified by LC-MS/MS }\end{array}$ & $\begin{array}{c}\text { Spectra counts } \\
\text { (IgG) }\end{array}$ & $\begin{array}{c}\text { Spectra counts } \\
\text { (V5) }\end{array}$ \\
\hline SMARCA4 & 0 & 17 \\
SMARCB1 & 1 & 3 \\
SMARCC1 & 0 & 6 \\
SMARCC2 & 0 & 10 \\
SMARCD2 & 2 & 14 \\
SMARCE1 & 0 & 6 \\
ACTL6A & 0 & 4 \\
\hline
\end{tabular}

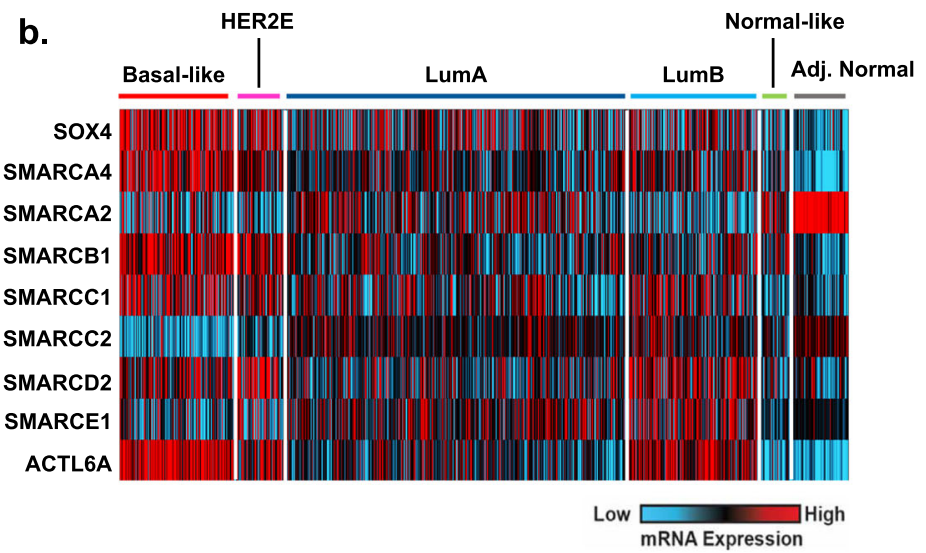

f.

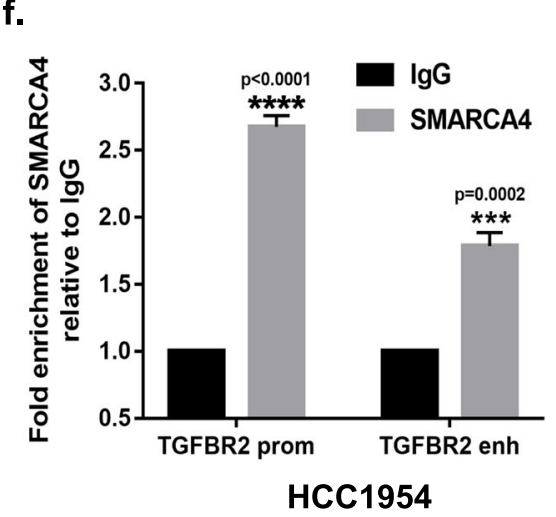

Fig. 5 SOX4 and SMARCA4 form a complex at TGFBR2 promoter and enhancer region. a Potential interacting partners of SOX4 from the SWI/SNF chromatin remodeling complex family of proteins identified by LC-MS/MS. Spectral counts for IgG and V 5 antibody for each protein is listed. b mRNA expression patterns of SWI/SNF complex core subunit genes relative to SOX4 were determined for 1031 human breast tumors and 94 adjacent normal samples from the TCGA dataset. Samples are organized by PAM50 molecular subtype; red indicates high mRNA expression, and blue depicts low mRNA levels. c Co- immunoprecipitation of SOX4 (V5) and SMARCA4 followed by reciprocal western blot analyses demonstrates the interaction between these proteins in HCC1143 ${ }^{\text {SOX4-V5 }}$ cells; results are representative of three independent experiments. d HEK293T cells were lentivirally overexpressed with HA-SOX4 and co-immunoprecipitation performed with HA antibody followed by western blot detection of SMARCA4 demonstrating the interaction between these proteins. e SMARCA4 ChIP-qPCR in HCC1143 demonstrated a 3.6- and 6.6-fold enrichment of SMARCA4 at the TGFBR2 promoter $(p=0.0024$; unpaired $t$-test) and enhancer ( $p=0.0008$; unpaired $t$-test) respectively. f SMARCA4 ChIP-qPCR in HCC1954 cells demonstrated a 2.7- and 1.8-fold enrichment of SMARCA4 at the TGFBR2 promoter $(p<0.0001$; unpaired $t$-test) and enhancer $(p=0.0002$; unpaired $t$-test), respectively.

correlation patterns were also observed between SOX4 and SWI/ SNF core subunits in the METABRIC dataset (Supplementary Fig. 6a). Given that SMARCA4, SMARCB1, SMARCC1, SMARCD2, and ACTL6A are highly expressed in basal-like tumors in TCGA and METABRIC datasets, our data suggest that the functional SWI/SNF complex may be significantly upregulated in these tumors and may interact with SOX4 to contribute to the aberrant signaling activity apparent in these tumors.

In order to confirm the interaction between SOX4 and SMARCA4, which is the catalytic subunit of the SWI/SNF chromatin remodeling complex, we performed co-immunoprecipitation (CoIP) experiments in $\mathrm{HCC} 1143^{\text {SOX4-V5 }}$ cells. As illustrated in Fig. $5 \mathrm{c}$, SMARCA4 was detected by western blot analyses following immunoprecipitation of SOX4 using an antibody against the V5 epitope tag. Importantly, reciprocal Co-IP experiments using the anti-SMARCA4 antibody followed by western blot analysis confirmed this interaction (Fig. 5c). Finally, to validate these findings in an independent cell line, using an independent epitope tag, HA-tagged SOX4 was lentivirally overexpressed in 293T cells. SOX4 was immunoprecipitated using an HA-specific antibody followed by SMARCA4 western blot analysis, which confirmed the interaction between SOX4 and SMARCA4 (Fig. 5d).
To determine if SMARCA4 is present at the TGFBR2 upstream regulatory regions, we analyzed publicly available SMARCA4 ChIPseq data ${ }^{51}$ from MDA-MB-231 cells. Concurrently, we analyzed publicly available ChIP-seq data for histone modifications that mark the promoter $(\mathrm{H} 3 \mathrm{~K} 4 \mathrm{me} 3)$ and enhancer $(\mathrm{H} 3 \mathrm{~K} 27 \mathrm{ac})$ regions to further characterize the TGFBR2 upstream regulatory regions ${ }^{52}$. These analyses identified increased enrichment of SMARCA4 at the TGFBR2 promoter and enhancer regions, indicated by the increased enrichment of H3K4me3 and H3K27ac histone marks, respectively (Supplementary Fig. 6b). Interestingly, SMARCA4 enrichment coincided with the SOX4-bound loci at the TGFBR2 promoter and enhancer regions that were previously identified (Fig. 4a) suggesting that SOX4 and SMARCA4 may form a complex at these sites. To validate this observation, SMARCA4 ChIP-qPCR analyses were performed in HCC1143 and HCC1954 cell lines using the previously described quantitative-PCR (qPCR) primers sets (Fig. 4). Our analyses demonstrated a significant 3.6-fold enrichment at the TGFBR2 promoter $(p=0.0024)$ and 6.6 -fold enrichment at the TGFBR2 enhancer region $(p=0.0008)$ relative to IgG in HCC1143 cells (Fig. 5e). These results were confirmed in the HCC1954 cell line, which showed a 2.6-fold and 1.7-fold enrichment of SMARCA4 at the TGFBR2 promoter $(p<0.0001)$ and enhancer $(p=0.0002)$ relative to lgG, respectively (Fig. $5 f$ ). 

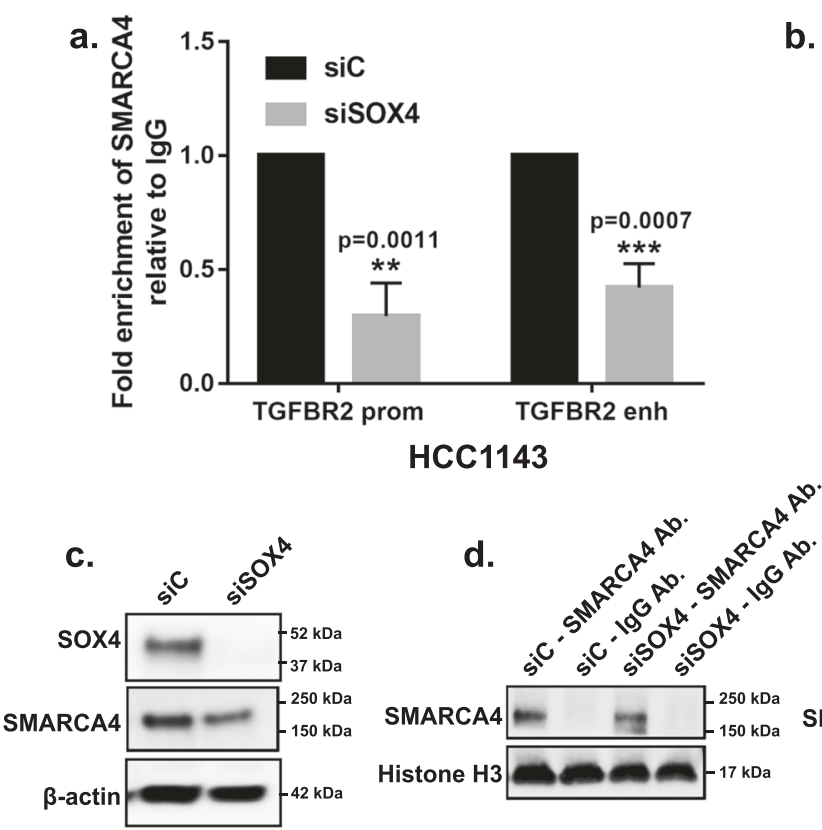

HCC1143

g.

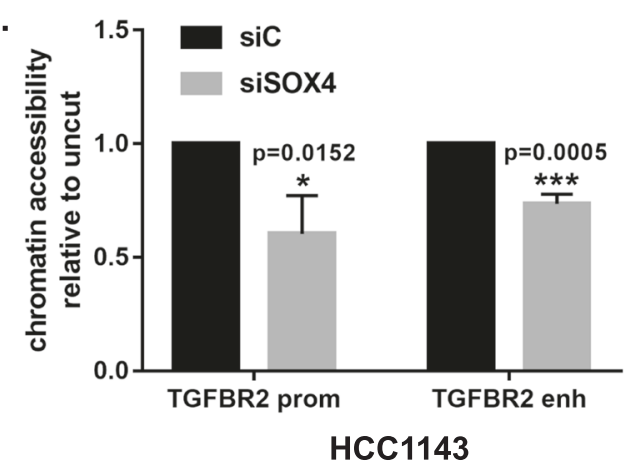

i.

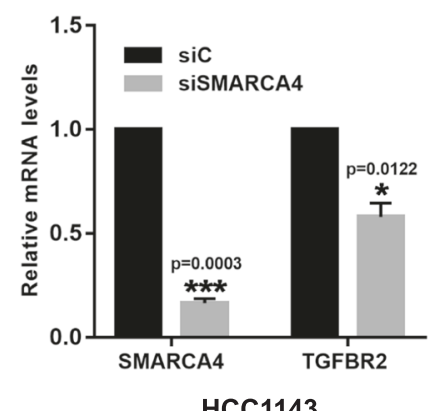

HCC1143 j.

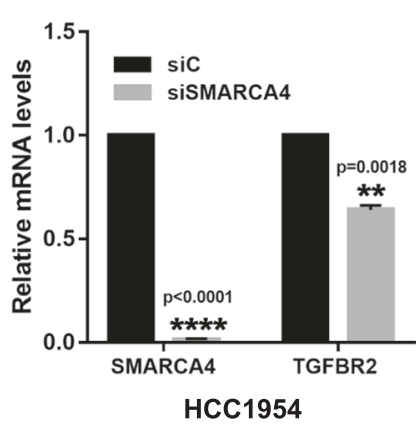

b.

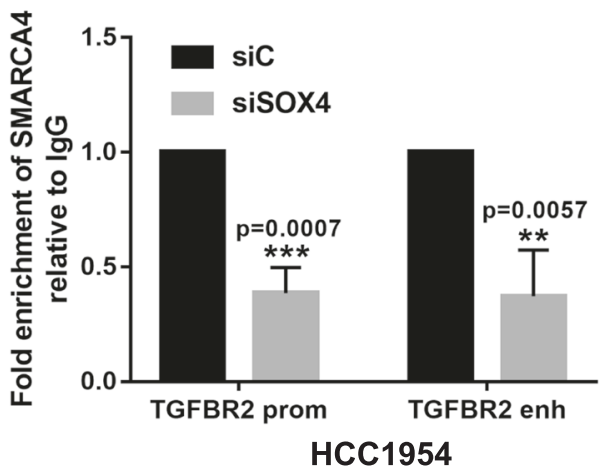

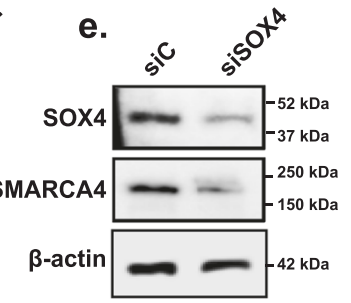

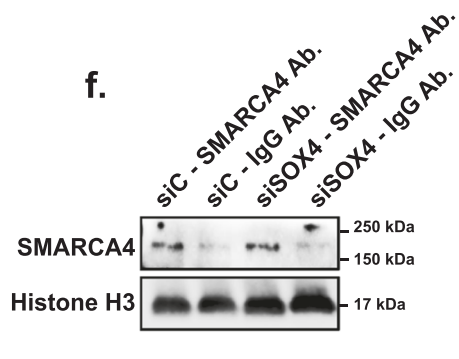

HCC1954

h.

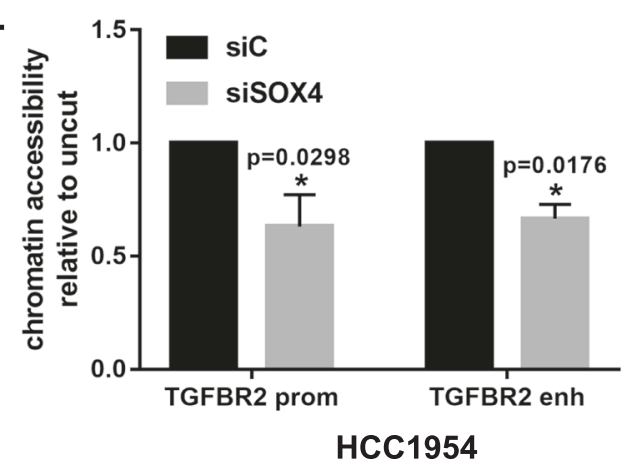

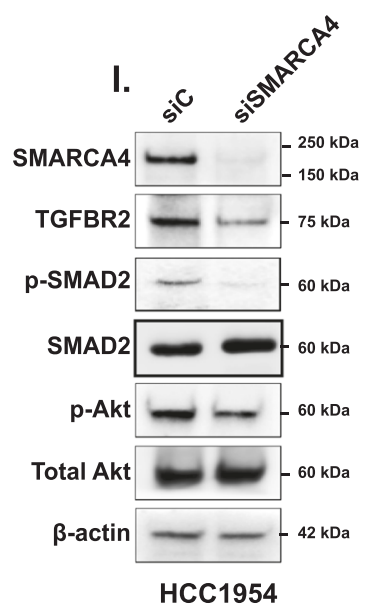

\section{SOX4 recruits SMARCA4 to regulatory regions to promote TGFBR2 expression}

SMARCA4 containing SWI/SNF complexes demonstrate little sequence specificity in vitro and are required to interact with transcriptional activator proteins in order to be recruited to the regulatory regions of target genes ${ }^{53,54}$. Given that our data indicate that SMARCA4 forms a complex with SOX4 at TGFBR2 regulatory regions, we next investigated whether SOX4 is required to recruit SMARCA4 to these regions.

To investigate this hypothesis, SMARCA4 ChIP-qPCR analyses were performed in the presence and absence of SOX4 in HCC1143 and HCC1954 cells. Our analyses determined that siRNA-mediated depletion of SOX4 in HCC1143 cell line resulted in a significant reduction in SMARCA4 enrichment on both the TGFBR2 promoter 
Fig. 6 SOX4 recruits SMARCA4 to remodel chromatin and mediates TGFBR2 expression. ChIP-qPCR demonstrating reduction in SMARCA4 enrichment following siRNA-mediated knockdown of SOX4 by $70.49 \%$ ( $p=0.001$; unpaired $t$-test) and $57.9 \%$ ( $p=0.0007$; unpaired $t$-test) in HCC1143 (a) and by $61.49 \%(p=0.0007$; unpaired $t$-test) and $62.8 \%(p=0.006$; unpaired $t$-test) in HCC1954 (b) cells at the TGFBR2 promoter and enhancer regions relative to the scrambled control. Western blot analysis demonstrating siRNA-mediated silencing of SOX4 results in decreased SMARCA4 protein expression in HCC1143 (c) and HCC1954 (e) cell lines. ChIP-western blot analysis demonstrating equivalent levels of SMARCA4 protein pulldown in cells treated with either siRNA targeting SOX4 or control siRNA in HCC1143 (d) and HCC1954 (f) cell lines. Chromatin accessibility assay demonstrating that accessibility was reduced following siRNA-mediated knockdown of SOX4 by $39.7 \%$ ( $p=$ 0.015 ; unpaired $t$-test) and $26.5 \%(p=0.0005$; unpaired $t$-test $)$ in HCC1143 (g) and by $36.9 \%(p=0.03$; unpaired $t$-test) and $33.4 \%(p=0.017$; unpaired $t$-test) in HCC1954 (h) cells at the TGFBR2 promoter and enhancer regions, respectively, relative to the scrambled control. qRT-PCR analysis demonstrating decreased TGFBR2 mRNA expression in HCC1143 (i) and HCC1954 (j) cell lines following RNAi-mediated silencing of SMARCA4. Representative western blot analysis demonstrating loss of TGFBR2 and pAkt protein expression in HCC1143 (k) and HCC1954 (I) cell lines following RNAi-mediated silencing of SMARCA4.

(70.39\% reduction, $p=0.001)$ and enhancer $(57.92 \%$ reduction, $p=0.0007$ ) regions relative to the scrambled control (Fig. 6a). As expected, similar results were observed in HCC1954 cells which showed a $61.5 \%$ decrease of SMARCA4 at the TGFBR2 promoter $(p$ $=0.0007)$ and $62.8 \%$ decrease at the TGFBR2 enhancer $(p=0.006)$ (Fig. 6b). We validated these findings in HCC1143 and HCC1954 cell lines which have been engineered to stably express one of two independent tet-inducible shRNA against SOX4. As illustrated in Supplementary Fig. 7, treatment with dox significantly abrogated the enrichment of SMARCA4 on the TGFBR2 promoter in both sh-1 (73.6\% reduction, $p=0.0002)$ and sh-2 $(56.1 \%$ reduction, $p=0.0005$ ) expressing HCC1143 cells (Supplementary Fig. 7a) as well as sh-1 (66.5\% reduction, $p=0.0014)$ or sh-2 $(56.0 \%$ reduction, $p=0.001$ ) expressing HCC1954 cells (Supplementary Fig. 7b). Similar results were observed for SMARCA4 enrichment at the TGFBR2 enhancer region with sh-1 expressing HCC1143 cells showing a $66.2 \%$ reduction, $(p=0.0004)$ and sh-2 expressing cells showing a $62.3 \%$ reduction $(p=0.002)$ relative to untreated cell lines (Supplementary Fig. 7a). Likewise, sh-1 (52.3\% reduction, $p=$ $0.02)$ or sh-2 $(60.6 \%$ reduction, $p=0.002)$ expressing HCC1954 cells showed a similar significant reduction in SMARCA4 enrichment at the TGFRB2 enhancer relative to untreated samples (Supplementary Fig. 7b).

Since previous studies have demonstrated that SOX4 can regulate SMARCA4 expression in MRC- 5 fibroblasts cells ${ }^{55}$, these data suggested the possibility that the observed decrease in SMARCA4 recruitment at the TGFBR2 promoter may be due to decreased SMARCA4 protein expression following SOX4 silencing. Given the noted tissue-specific nature of SOX4 gene expression programs, we next assessed whether SOX4 could mediate expression of SMARCA4 in TNBC cell lines ${ }^{56}$. As illustrated in Fig. $6 \mathrm{c}$ (HCC1143) and Fig. 6e (HCC1954), siRNA-mediated silencing of SOX4 resulted in decreased SMARCA4 protein expression in both cell line models. To determine if the observed decrease in SMARCA4 expression was sufficient to explain the loss of SMARCA4 binding to the TGFBR2 regulatory regions, we performed ChIP followed by western blot analysis in siControland siSOX4-treated cells. These analyses detected equivalent SMARCA4 levels in both cell lines (Fig. 6d, f) demonstrating that an equal amount of chromatin bound SMARCA4 could be detected following siSOX4 or siControl treatment. These data indicate that while loss of SOX4 decreases overall SMARCA4 expression, it does not globally affect its ability to bind to DNA. Importantly, however, loss of SOX4 does affect SMARCA4 recruitment to the TGFBR2 promoter/enhancer indicating that this process is dependent on the presence of SOX4 at these regions. Moreover, these analyses also suggest that SOX4 mediates SMARCA4 activity at both the transcript and protein levels and may further explain why there is a strong correlation between SOX4 and SMARCA4 expression in human tumors (Fig. 5b and Supplementary Fig. 6a).

Next, we examined whether SOX4-mediated recruitment of SMARCA4 to the TGFBR2 promoter and enhancer region is a silent or a functional recruitment. SMARCA4 is the catalytic subunit of the SWI/SNF complex and is required for chromatin remodeling to increase accessibility at genomic $\operatorname{loci}^{47}$. To assess the remodeling at the TGFBR2 regulatory regions mediated by the SOX4-SMARCA4 interaction, we performed a micrococcal nuclease (MNase) digestion assay followed by $\mathrm{APCR}^{48}$ following siRNA or shRNA depletion of SOX4. We determined that siRNA-mediated silencing of SOX4 resulted in a significant $39.7 \%(p=0.015)$ and $26.5 \%(p=$ $0.0005)$ reduction in chromatin accessibility the TGFBR2 promoter and enhancer regions, respectively, relative to the scrambled control in HCC1143 (Fig. 6g). A similar 36.9\% $(p=0.03)$ and $33.4 \%$ $(p=0.018)$ reduction in chromatin accessibility was observed at the promoter and enhancer regions in HCC1954 cells following siRNA-mediated depletion of SOX4 (Fig. 6h). As expected, comparable results were observed after dox induced knockdown of SOX4 at the TGFBR2 promoter in sh-1 $(37.1 \%$ reduction, $p=$ $0.03)$ or sh-2 (35.6\% reduction, $p=0.004)$ expressing HCC1143 (Supplementary Fig. 7c) as well as sh- 1 (82.1\% reduction, $p=0.03$ ) or sh-2 (99.8\% reduction, $p<0.0001)$ expressing HCC1954 cells (Supplementary Fig. 7d). Similarly, we observed a $27.2 \%$ and $29.3 \%$ reduction in chromatin accessibility at the TGFBR2 enhancer in sh-1 $(p=0.022)$ or sh-2 $(p=0.02)$ expressing HCC1143 cells, respectively, relative to untreated cells (Supplementary Fig. 7c). Likewise, sh-1 $(59.6 \%$ reduction, $p=0.01)$ or sh-2 $(95.1 \%$ reduction, $p<0.0001)$ expressing HCC1954 cells showed a significant reduction in chromatin accessibility at the TGFRB2 enhancer relative to control cells (Supplementary Fig. 7d).

Collectively, these data suggest that SOX4 is required to recruit SMARCA4 to TGFBR2 regulatory regions and that SMARCA4 is essential for chromatin remodeling and activation of TGFBR2 expression. Consistent with the latter premise, qRT-PCR and western blot analysis demonstrated a significant reduction of TGFBR2 mRNA (HCC1143, $p=0.012$; HCC1954, $p=0.001$ ) and protein (HCC1143, $p=0.0009$; HCC1954, $p<0.0001$ ) levels following siRNA-mediated silencing of SMARCA4 in HCC1143 (Fig. 6i, k and Supplementary Fig. 7f) and HCC1954 (Fig. 6j, I and Supplementary Fig. 7g) cell lines. As expected, Akt (Ser473) phosphorylation was significantly reduced in HCC1143 (Fig. 6k and Supplementary Fig. 7f) $(p=0.0037)$ and HCC1954 (Fig. $6 \mathrm{I}$ and Supplementary Fig. $7 \mathrm{~g})(p=0.0002)$ cells following siSMARCA4 treatment compared to siControl-treated cells. These data suggest that SOX4-mediated recruitment of SMARCA4 on TGFBR2 regulatory regions results in a more open and permissive chromatin conformation, resulting in increased TGFBR2 expression and downstream activation of PI3K signaling.

\section{DISCUSSION}

TNBC accounts for 10-15\% of new breast-cancer cases and $~ 1$-in-4 breast-cancer-related deaths each year ${ }^{1,2}$. Although a number of recent advances have improved treatment strategies, there is a clear need to better understand the mechanisms driving TNBC genesis and progression in order to develop more effective therapies. Despite the fact that basal-like or triple-negative breast tumors are characterized by aberrant activation of PI3K/Akt signaling, PI3K-family inhibitors have shown limited clinical 

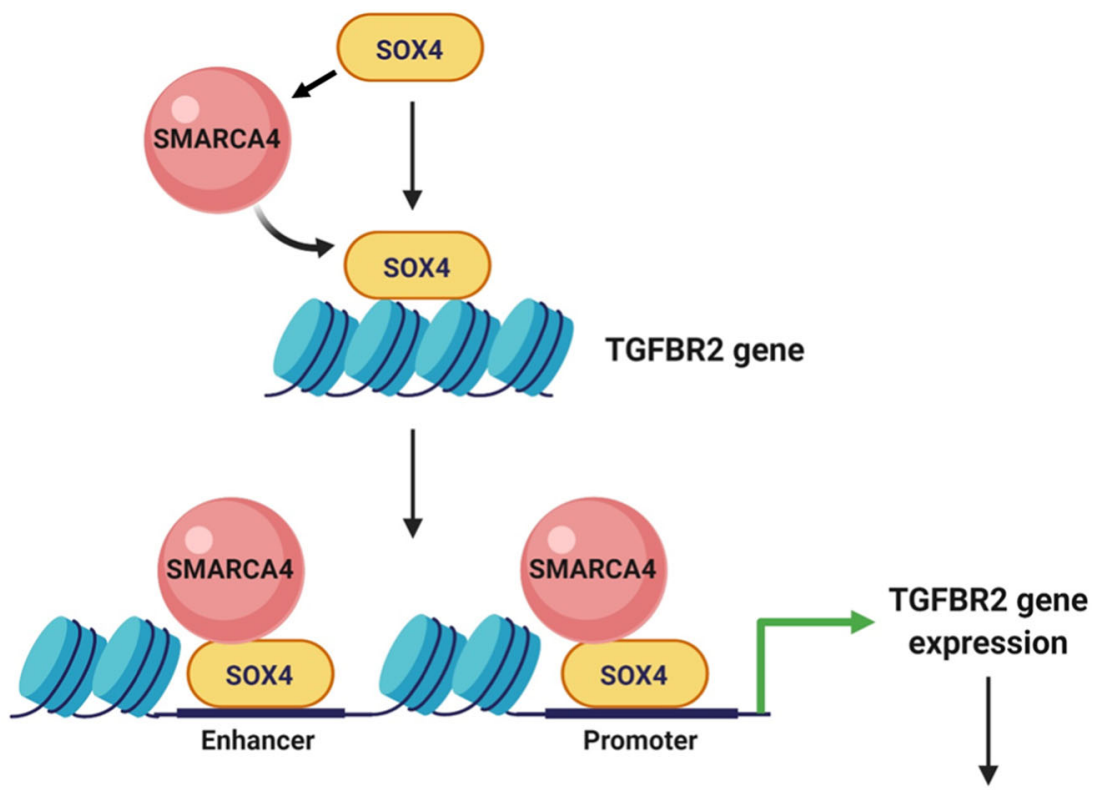

PI3k/Akt signaling activation

Fig. 7 The Sox4-SMARCA4 complex mediates PI3K/Akt signaling by regulating TGFBR2 gene expession. Schematic representation of the hypothetical model demonstrating SOX4 and SMARCA4-mediated regulation of the TGFBR2 gene expression and activation of PI3K/Akt signaling.

success either as single agent therapies or as combination therapies in this subset of patients ${ }^{7,12,14}$. The lack of clinical response can be attributed to a number of factors, including, but not limited to, the development of adaptive resistance mechanisms and/or activation of parallel signaling pathways $s^{7,12,14}$. These previous studies suggest that understanding and targeting additional mechanisms of the PI3K pathway activation and/or complementary pathways will be important for optimizing therapeutic strategies for TNBC patients. While several largescale genomic studies have identified copy-number alterations or mutations in key regulatory components of the PI3K pathway in specific subsets of TNBC or basal-like tumors ${ }^{3}$, there is a significant need to understand additional mechanisms regulating $\mathrm{PI} 3 \mathrm{~K}$ signaling and to identify key genes that mediate the development of adaptive responses to PI3K pathway inhibitors in these patients.

A recent genomics-based study of human tumors from our laboratory identified SOX4 as a potential driver of PI3K/Akt signaling in basal-like breast cancer and in vitro studies confirmed that SOX4 can regulate this pathway in basal-like cell lines ${ }^{5}$. In the current study, we identified a novel mechanism by which SOX4 regulates $\mathrm{PI} 3 \mathrm{~K}$ signaling in basal-like breast cancer. Using a combination of proteomics-based kinome profiling, transcriptomic analyses and molecular approaches, we identified TGFBR2 as a critical downstream target of SOX4 in basal-like breast-cancer cell lines. Importantly, in vitro analyses confirmed that SOX4-mediated activation of PI3K signaling is regulated by TGFBR2 activity. Mechanistic studies established direct binding of SOX4 to the TGFBR2 promoter and enhancer regions indicating that TGFBR2 is a direct transcriptional target of SOX4. Finally, we demonstrated that SOX4 recruits and forms a complex with the SMARCA4 at the TGFBR2 promoter and enhancer regions. This complex is required to mediate chromatin remodeling and facilitate activation of TGFBR2 expression and downstream PI3K signaling (Fig. 7). Therefore, the current study not only delineates a novel mechanism by which SOX4 cooperates with SMARCA4 to regulate PI3K signaling through TGFBR2 expression but these findings also have a potentially broader implication for regulation of oncogenic signaling and resistance mechanisms in TNBC.
SOX4 is a well-established oncogene and a member of SOX C family of SRY-related HMG-box (SOX) transcription factors ${ }^{16}$. Increased SOX4 expression has been shown to be associated with malignant transformation and metastasis in several cancer types $^{21-24}$, including breast cancer ${ }^{5,17-20}$. Despite these findings, a limited understanding of the mechanisms, including gene expression programs mediated by SOX4 and necessary co-factors, have been defined in basal-like or TNBCs. Analyses of human breast tumors indicate that SOX4 mRNA is uniformly overexpressed in basal-like tumors relative to adjacent normal tissue along with the SWI/SNF catalytic subunit SMARCA4 and SMARCB1 $S M A R C C 1, S M A R C D 2$, and ACTL6A, which encode for the essential subunits of this complex (Fig. $5 \mathrm{~b}$ ). Our data further demonstrate that SMARCA4 and SOX4 form a novel complex to mediate TGFBR2 expression and PI3K signaling and that inhibition of either gene results in a similar effect on downstream signaling. These results are consistent with the reported pro-oncogenic role for SWI/SNF complex proteins, and in particular the role of SMARCA4 in regulating oncogenic phenotype in breast cancer ${ }^{28,29,34,35,48,57}$. As such, our data indicate that the SOX4-SMARCA4 complex may play an integral role in TNBC genesis and progression by activation of novel gene expression programs in this subset of breast tumors.

TGFBR2 is a transmembrane serine/threonine protein kinase that activates canonical SMAD signaling following heterodimerization with TGFBR1 in a ligand-dependent or independent manner ${ }^{58}$. While, it is unclear whether SOX4 activation of PI3K signaling is ligand-dependent, it has been documented that the TNBC tumor microenvironment is often enriched with TGF $\beta$-family ligands produced by tumor cells and/or tumor-associated stromal and immune cells ${ }^{59,60}$. However, since aberrantly, or constitutively, activated TGFBR2 can also modulate TGF $\beta$ signaling independent of ligand, additional studies will be required to further delineate this process in SOX4 overexpressing human tumors. Nonetheless, the impact of TGF $\beta$ signaling on breast-cancer genesis and progression, including its role in EMT and proliferation, has been well-documented $^{17,19,20}$. In addition to canonical signaling, TGFBR2 can also regulate noncanonical signaling through activation of PI3K, MAPK, and WNT signaling ${ }^{61}$. While previous studies 
have indicated that cross-talk between SOX4 and TGF $\beta$ can mediate activation of canonical Smad signaling in order to induce $\mathrm{EMT}^{17,19,20}$, the role of SOX4 in regulating noncanonical TGF $\beta$ signaling in cancer, or in TNBC or basal-like breast cancer specifically, has not been reported. TGFBR2 has been shown to stimulate noncanonical $\mathrm{PI} 3 \mathrm{~K}$ signaling by interacting with and phosphorylating the p85 regulatory subunit of PI3 kinase in fibroblast cell lines ${ }^{62}$. Therefore, the observation that SOX4 and SMARCA4 mediate PI3K activity through TGFBR2 expression identifies a novel and direct role for TGFBR2 in activating noncanonical PI3K signaling in basal-like breast cancer. Furthermore, these findings may also suggest that additional oncogenic signaling pathways, downstream of TGF $\beta$, may be activated by the SOX4/SMARCA4 complex and contribute to tumor development and progression. Consistent with this idea, previous studies have indicated that increased TGFBR2 expression is associated with resistance to ALK and EGFR inhibitors in non-small cell lung carcinoma. In these studies, TGFBR2 was shown to mediate resistance by activating noncanonical MEK-ERK signaling ${ }^{63}$. Similarly, in breast cancer, autocrine activation of TGFBR2 expression in response to chemotherapy has been reported to regulate proliferation of breast-cancer stem cell populations and contribute to tumor recurrence ${ }^{59}$. Given that our data identifies SOX4 and SMARCA4 as key modulators of TGFBR2 expression and PI3K activity in basal-like breast cancer, it remains to be determined if aberrant TGFBR2 activity can also regulate a similar resistance mechanism to $\mathrm{PI} 3 \mathrm{~K}$-family inhibitors in TNBC patients.

Profiling of the drug-able kinome by MIB/MS analysis identified a number of kinases that were significantly depleted or activated following SOX4 knockdown. Importantly, TGFBR1, which forms a functional complex with TGFBR2, and EPHA4, a known downstream target of TGF- $\beta$ signaling that is associated with poor prognosis in basal-like breast-cancer patients ${ }^{64}$, were downregulated in MIB/MS analysis. These findings along with depleted p-SMAD2 protein levels upon SOX4 knockdown indicates that SOX4-mediated activation of TGFBR2 could potentially regulate both canonical and noncanonical TGF- $\beta$ signaling pathways. Moreover, in addition to TGFBR2 and EPHA4, we identified several kinases (BMPR1A, ACVR1B, LCK, and EGFR), which have been shown to activate PI3K activity to be downregulated upon SOX4 knockdown, suggesting that SOX4 may mediate activation of $\mathrm{PI} 3 \mathrm{~K} / \mathrm{Akt}$ signaling through multiple mechanisms and/or may mediate complementary pathways that contribute to resistance to PI3K-family drugs. Consistent with this premise, MIB/MS analyses also identified significant enrichment of MAPK signaling pathway components MAPK1, MAP2K1 (MEK1), and MAP2K4 (MEK4) following siRNA-mediated SOX4 silencing. Activation of MAPK signaling has been identified as a feedback mechanism to compensate for the downregulation of $\mathrm{PI} 3 \mathrm{~K}$ signaling in breastcancer patients treated with everolimus in ER+ breast cancers ${ }^{65}$. This suggests that enrichment of these MAP kinases could function as compensatory mechanism to PI3K inhibition and dual targeting of these pathways may yield a more durable response in TNBC patients. In agreement with this notion, a phase 1 clinical trial investigating co-targeting of $\mathrm{PI} 3 \mathrm{~K}$ and MAPK signaling pathways showed a durable response in KRAS-driven tumors of colon, pancreas, skin, and lung ${ }^{66}$, suggesting that targeting these pathways together may prove beneficial in TNBC patients. Moreover, several clinical trials are currently underway to test the efficacy of targeting these pathways together in TNBC patients ${ }^{67}$. Taken together, these data in combination with transcriptome profiling by RNAseq, the identification of the SOX4 interactome by quantitative MS/MS and SOX4-mediate kinome by MIB/MS analysis suggest that additional signaling pathways that contribute to aspects of tumor progression may be regulated by SOX4 alone or in concert with SMARCA4 in basal-like tumors.
The current study has identified a novel mechanism by which PI3K signaling is regulated in basal-like or TNBC. Our data demonstrate that SOX4 and SMARCA4, which are uniformly overexpressed in basal-like tumors, form a previously unreported complex that is required to mediate epigenetic reprogramming of the TGFBR2 chromatin landscape and regulate TGFBR2 expression to promote PI3K signaling in this subset of breast-cancer patients. Profiling of the drug-able kinome in addition with transcriptome analyses suggest that SOX4 alone and in combination with SMARCA4 may modulate unique gene expression programs that contribute to breast-cancer genesis, progression, and response to therapy. Additional studies will be required to delineate these signaling networks, their impact on tumorigenesis and response to therapies, including resistance to PI3K-family inhibitors in TNBC patients, and to determine whether these networks are active in other breast-cancer subtypes and/or other forms of cancer.

\section{METHODS}

Gene expression analysis of human breast tumors and breastcancer cell lines

RNA sequencing data from human tumors $(n=1031)$ and adjacent normal tissue $(n=94)$ were acquired from the TCGA data portal (https://tcga-data. nci.nih.gov/tcga/), RSEM values were log-transformed and mediancentered, and genes were filtered to exclude those that were not present in at least $80 \%$ of samples ${ }^{68}$. Illumina HT-29 v3 expression data for the METABRIC project $(n=1992)$ was acquired from the European Genomephenome Archive at the European Bioinformatics Institute (https://www. ebi.ac.uk/ega/) and data were median-centered ${ }^{36}$. PAM50 classification has been previously reported for the TCGA and METABRIC datasets; these data are summarized in Supplementary Data 1 and $2^{5,36}$. The PI3K pathway gene expression signature score was calculated for each tumor sample in the TCGA and METABRIC dataset as previously reported ${ }^{39}$. Briefly, gene expression data for each dataset were filtered to those genes present in the PI3K signature and the pathways score determined for each sample by calculating the mean value of the signature genes. The signature score is reported for each sample in each dataset (Supplementary Data 1 and 2). To examine the relationship between SOX4 mRNA expression and PI3K activity, samples in each dataset were delineated into quartiles, and PI3K pathway score in the top and bottom quartiles was assessed by a twotailed $t$-test. Relative SOX4 expression between basal-like tumors $(n=179)$ and adjacent normal $(n=94)$ samples from the TCGA dataset was compared by a two-tailed $t$-test. For analyses of individual gene expression patterns relative to PAM50 subtype, expression values for specific genes were selected from the TCGA or METABRIC dataset. Samples were categorized by PAM50 subtype, differences in subtype-specific expression determined by ANOVA test followed by Tukey test for pairwise comparison, and relative expression patterns visualized by the "imagesc" function in MATLAB. To identify basal-like breast-cancer cell lines with high SOX4 expression for mechanistic studies, SOX4 mRNA and protein expression was assessed in a panel of basal-like cell lines. Affymetrix $\mathrm{U} 133+2$ gene expression data for a panel of 24 basal-like breast-cancer cell lines were acquired from Gene Expression Omnibus (GEO) (GSE12777) ${ }^{40}$, data were MAS5-normalized and log2-transformed using Affymetrix Gene Expression Console, and gene-specific expression probes were collapsed to the median using GenePattern ${ }^{69}$ and $z$-score reported. MS-derived protein expression values for nine basal-like breast-cancer cell lines were acquired from the CCLE project ${ }^{70}$ and $z$-score reported.

\section{Cell culture and shRNA/siRNA knockdown}

Breast-cancer cell lines and HEK293T cells were purchased from the American Tissue Culture Collection (Manassas, VA, USA) and cultured according to the suggested guidelines. HCC1143 or HCC1954 cell lines expressing one of the two tetracycline (tet)-inducible shRNA expressing cell lines were created using the pTRIPZ Inducible Lentiviral shRNA system (GE Dharmacon). The catalog number for shRNA (1) is: V2THS_153343 and for shRNA (2) is: V3THS_401094. The shRNA expression was induced using $2.0 \mu \mathrm{g} / \mathrm{mL}$ of dox for $\mathrm{HCC} 1143$ and $1.0 \mu \mathrm{g} / \mathrm{mL}$ of dox for HCC1954. Silencing of SOX4 expression was verified by qRT-PCR analysis. For siRNA knockdown experiments, cells were subcultured at $60-70 \%$ confluence before transfections. Lipofectamine RNAiMAX (ThermoFisher) was used to transfect cells with $50 \mathrm{~nm}$ of SMART pool siRNA targeting either SOX4 (L- 
011779-00-0005), SMARCA4 (L-010431-00-0005), TGFBR2 (LQ-003930-000005), or non-targeting siRNA control pool (D0012061305) according to manufacturer's instructions (Dharmacon) for $96 \mathrm{~h}$ prior to harvesting cells for subsequent experiments.

\section{RNA extraction and quantitative real-time PCR}

Total RNA was isolated using the RNeasy plus Mini Kit (Qiagen), and CDNA was synthesized using the QuantiTect Reverse Transcription kit (Qiagen). qPCR was performed and analyzed using Applied Biosystems QuantStudio3 real-time thermal cycler system. Primer sequences are as follows: human SOX4: Forward: 5'-CTCTCCAGCCTGGGAACTATAA-3', Reverse: 5'-CG GAGGTGGGTAAAGAGAGAA-3'; human SMARCA4: Forward: 5'- AGTGCTGCT GTTCTGCCAAAT - $3^{\prime}$, Reverse: 5'-GGCTCGTTGAAGGTTTCAG-3'; human TG FBR2: Forward: 5'- AAATGGAGGCCCAGAAAGAT-3', Reverse: 5'- ACTTGACTG CACCGTTGTTG-3'; and human GAPDH: Forward: 5'- TCTGACTTCAACAGCGA CAC-3', Reverse: 5'-CCAGCCACATACCAGGAAAT-3'.

\section{Cell extracts and western blot analysis}

Cells were harvested using Triton lysis buffer (TLB) containing 25-mM HEPES, 100-mM NaCl, 1-mM EDTA, $10 \%$ glycerol, and 1\% Triton X-100 with protease and phosphatase inhibitor added fresh prior to use. Protein concentrations were measured using the BCA protein assay kit and $35-50 \mu \mathrm{g}$ of protein was loaded on 4-20\% Mini-protean TGX gradient gel (BioRad) at $100 \mathrm{~V}$ for $2 \mathrm{~h}$ at room temperature and transferred onto nitrocellulose membrane at $35 \mathrm{~V}$ for $2 \mathrm{~h}$ at $4{ }^{\circ} \mathrm{C}$. The membranes were blocked using AdvanBlock-chemi blocking solution (Advansta) for $1 \mathrm{~h}$ at room temperature, incubated with primary antibody (1:1000) overnight at $4{ }^{\circ} \mathrm{C}$ followed by incubation with HRP-conjugated secondary antibodies (1:2000, Cell Signaling Technology) for $1 \mathrm{~h}$ at room temperature. The signal was developed using SuperSignal West Pico Chemiluminescent Substrate (ThermoFisher Scientific) and digitally imaged using the ChemiDoc Touch Imaging System (BioRad). Primary antibodies against pAkt (S473) (4060S), Total Akt (9272S), pP70S6K (Thr389) (9205S), P70S6K (9202S), p4EBP1 (Thr37/46) (2855S), 4EBP1 (9452S), p-SMAD2 (3108S), SMAD2 (3122 S), V5 (13202S), HA (3724S), $\beta$-actin (4970S) were purchased from Cell Signaling Technology. The antibodies against SOX4 (PB9618, BosterBio), SMARCA4 (sc-374197, Santa Cruz Biotechnology), and TGFBR2 (AF-241-SP, R\&D Biosystems) were used according to the instructions of the manufacturer. All western blots presented in a given figure panel are derived from a single experiment and the full uncropped images are presented in Supplementary Fig. 8; a minimum of three independent experimental replicates were performed for each analysis.

\section{CRISPR-Cas9-mediated tagging of SOX4 locus with V5 epitope}

The SOX4 locus was tagged with a V5 epitope at the C-terminus in HCC1143 cells using CRISPR-Cas9-mediated knock-in strategy. Briefly, a duplex oligo containing gRNA C187 was cloned into pSpCas9 (BB)-2A-GFP (pX458), which was a gift from Dr Feng Zhang (Addgene \# 48138) to create plasmid $\mathrm{pX} 458-\mathrm{C} 187$. The donor oligo containing the $\mathrm{V} 5$ tag sequence was ordered from IDT, PAGE purified, and $0.5 \mu \mathrm{g}$ of donor oligo was cotransfected with $2 \mu \mathrm{g}$ of pX458-C187 into $2 \times 106 \mathrm{HCC} 1143$ cells using the NEON transfection system. Twenty-four hours post-transfection, $0.5 \times 106$ GFP-positive cells were individually sorted into seven 96-well plates from which 32 clones grew and were genotyped using the following primers, SOX4A: 5'-AAGACGACCTGCTCGACCTGAAC-3'; SOX4B: 5'-CTTGATCCGACGACGAGAACGC- $3^{\prime}$. Ten clones were sequenced by the Sanger method and two clones, which had a complete V5 tag inserted correctly at the $C$ terminus, were expanded into cell lines. The gRNA and oligo donor sequence used are listed below:

gRNA sequence and location:

CGAGTCCAGCATCTCCAACC TGG [GRCh38.p12 Chrom.6: 2159592321595945(+)]

Donor oligo sequence $[(-)$ strand]:

$5^{\prime}$... TCACTIIIIIITCTCCTCTCCTACCCCCCCCGGCCCTTCTCCCTGCCTGC GCGCCCTtcaCGTAGAATCGAGACCGAGGAGAGGGTTAGGGATAGGCTTACCG TAGGTGAAAACGAGGTTGGAGATGCTGGACTCGAGCCAGTCTCCCGAGATC ATCTCGCTCAC...3'

V5 tag, underlined; altered PAM motif, double underlined; stop codon, lower case; gRNA sequence, bold.

\section{Immunofluorescence}

Immunofluorescence studies were performed as described previously ${ }^{5}$. Briefly, cells were grown on coverslips, fixed with $4 \%$ paraformaldehyde in phosphate-buffered saline (PBS), and permeabilized with $0.25 \%$ Triton X100 in PBS for $8 \mathrm{~min}$ at room temperature. Cells were blocked in 1\% BSA in PBS with $0.05 \%$ Tween 20 for 45 min at room temperature, incubated with V5 antibody for $1 \mathrm{~h}$ at room temperature, and then incubated with fluorochrome-labeled secondary antibody for $1 \mathrm{~h}$ at room temperature. The coverslips were counterstained with DAPI and imaged with a Nikon Eclipse TE-2000U fluorescent microscope.

\section{Co-IP and LC-MS/MS analysis}

The HA epitope-tagged SOX4 plasmid was generously provided by $\mathrm{Dr}$ Carlos Moreno (Emory University) and purified to generate lentiviral particles for transduction of HEK293T cells. Protein lysates were prepared from either HCC1143 $3^{\text {SOX4-V5 }}$ or HA-SOX4-transduced HEK293 cells and CoIP studies were performed as described previously ${ }^{71}$. Briefly, cells were washed two times with PBS and lysed in TLB buffer using diagenode bioruptor. Lysate was then centrifuged at $13,000 \times g$ for $15 \mathrm{~min}$. Protein concentrations were measured using the BCA protein assay kit and $2000 \mu \mathrm{g}$ of protein lysate was incubated with $5 \mu \mathrm{g}$ of antibody against HA, V5, or SMARCA4, or with a non-specific lgG antibody overnight at $4^{\circ} \mathrm{C}$, followed by the addition of protein $G$ Dynabeads (Thermofisher \#10004D) and an additional incubation while rocking for $2 \mathrm{~h}$ at $4{ }^{\circ} \mathrm{C}$. Beads were washed three times in the lysis buffer, eluted with $2 X$ SDS gel loading buffer, and analyzed by western blotting.

For LC-MS/MS analysis, $9000 \mu \mathrm{g}$ of HCC1143 SOX4-V5 protein lysate was used to precipitate the protein complexes using either $5 \mu \mathrm{g}$ of $\mathrm{V} 5$ antibody or a non-specific lgG control as described above. The precipitated protein complexes were separated on a 4-20\% gradient SDS gel, visualized using the SYPRO Ruby protein gel staining kit (Thermofisher \#S12000) and analyzed on Thermo Scientific Orbitrap Fusion Lumos Tribrid mass spectrometer. Briefly, each gel lane was cut, in-gel trypsin digested (protein/trypsin $\sim 50: 1$ ) at $37^{\circ} \mathrm{C}$ for $16 \mathrm{~h}$. The tryptic peptides were extracted and desalted using a C-18 cartridge prior to LC-MS/MS analysis. For LC-MS/MS, the peptides were separated on a C-18 nano column $(75 \mu \mathrm{m} \times 50 \mathrm{~cm}, 2 \mu \mathrm{m}, 100 \AA)$ at a flow rate of $300 \mathrm{~nL} / \mathrm{min}$ on an UltiMate 3000 LC system using a $2 \mathrm{~h}$ gradient of solvent $A$ ( $2 \%$ acetonitrile in $0.1 \%$ $\mathrm{FA})$ and solvent $\mathrm{B}(85 \%$ acetonitrile in $0.1 \% \mathrm{FA})$. The MS/MS spectra were acquired on an Orbitrap Fusion Lumos mass spectrometer (Thermo Scientific, Canoga Park, CA) using the data-dependent analysis mode. MS scan range is $m / z 375-1500$ with the resolution of 120,000 (FWHM). The capillary temperature was set to $275^{\circ} \mathrm{C}$, and the spray voltage set to $2 \mathrm{kV}$. The MS/MS spectra were searched against the Uniprot human database (74,072 entries) using the Sequest search engine through the Proteome Discoverer (Version 2.3) platform. The mass tolerance was $10 \mathrm{ppm}$ for MS and $0.1 \mathrm{Da}$ for MS/MS. The variable modifications included methionine oxidation and protein $\mathrm{N}$-terminus acetylation, whereas the fixed modification included cysteine carbamidomethylation. All proteins and peptides were identified with a false discovery rate of $<1 \%$. Relative quantitation was calculated based on a spectral counting method, where the spectral counts of identified proteins in V5 pulldown were divided by their corresponding spectral counts in non-specific lgG control. To avoid a large ratio from the presence of zero in the denominator, we arbitrarily added two spectral count to both the numerator and denominator during the ratio calculation.

\section{TGFBR2 overexpression and rescue experiments}

pHAGE-TGFBR2 plasmid was a gift from Drs Gordon Mills and Kenneth Scott (Addgene plasmid \# 116800). Lentiviral particles were generated by co-transfecting pHAGE-TGFBR2 plasmid and the packaging plasmids (psPAX2 and pMD2.G) in 293T packaging cell line using Lipofectamine 2000 transfection reagent. Viral supernatants were collected at 48 and $72 \mathrm{~h}$, centrifuged to remove cell debris, filtered through $0.45-\mu \mathrm{m}$ filters (Corning) and viral particles quantified using the Lenti-X GoStix Plus kit. The purified pHAGE-TGFBR2 viral particles were then used to perform overexpression and rescue experiments by infecting $(\mathrm{MOI}=3) \mathrm{HCC} 1143$ and HCC1954 cells that have been transfected with siRNA targeting either SOX4 or non-targeting control siRNA for $96 \mathrm{~h}$. Cells were harvested for protein analysis $48 \mathrm{~h}$ post overexpression. 


\section{Kinome profiling by MIB/MS}

For MIB affinity chromatography, HCC1143 cells treated with either siRNA targeting SOX4 or non-targeting control siRNA for $96 \mathrm{~h}$ were washed $1 \mathrm{X}$ with cold PBS, lysed in 50-mM HEPES, $150-\mathrm{mM} \mathrm{NaCl}, 0.5 \%$ Triton X-100, 1mM EDTA, 1-mM EGTA, at pH 7.5 containing 10-mM NaF, 2.5-mM NaVO4, Complete protease Inhibitor Cocktail (Roche), and 1\% Phosphatase Inhibitor Cocktails 2 and 3 (Sigma). Lysate was sonicated three times $(10 \mathrm{~s})$ on ice and centrifuged (10 min, $13,000 \mathrm{rpm})$ at $4^{\circ} \mathrm{C}$ and the supernatant syringe-filtered through a 0.2-mM SFCA membrane. Lysate was equalized at $500-\mu \mathrm{g}$ total protein per experiment to $1-\mathrm{M} \mathrm{NaCl}$ and flowed over kinase inhibitor bead resin, a $175-\mu \mathrm{L}$ mixture of five kinase inhibitors (VI-16832, PP58, Purvalanol B, UNC-21474, and BKM-120) custom-synthesized with hydrocarbon linkers (except Purvalanol B) and covalently linked to ECH-Sepharose (or EAH-Sepharose for Purvalanol B) beads as previously described ${ }^{41}$, followed by 30 volumes of washes with high salt $(1-\mathrm{M} \mathrm{NaCl})$ and low salt $(150-\mathrm{mM} \mathrm{NaCl})$ buffer (50-mM HEPES, $0.5 \%$ Triton X-100, 1-mM EDTA, 1-mM EGTA, at pH 7.5). Bound kinases were eluted by boiling with $0.5 \%$ SDS and $1 \% \beta$-mercaptoethanol in $100-\mathrm{mM}$ Tris- $\mathrm{HCl}, \mathrm{pH} 6.8,2 \times 15 \mathrm{~min}$, treated with $\mathrm{DTT}\left(5 \mathrm{mM}, 25 \mathrm{~min}\right.$ at $\left.60^{\circ} \mathrm{C}\right)$ and lodoacetamide ( $20 \mathrm{mM}, 30 \mathrm{~min}$ in the dark at RT), and spin-concentrated to $100 \mu \mathrm{L}$ (Amicon Millipore Amicon Ultra-4, 10-K cutoff) before Methanol/ Chloroform precipitation. Proteins were trypsinized overnight at $37^{\circ} \mathrm{C}$, extracted with water-saturated ethyl acetate four times, then dried down in a speed-vac. Peptides were desalted with C-18 spin columns (Cat. 89870, Pierce) according to the manufacturer's instructions.

Peptides were resuspended in $0.1 \%$ formic acid. Forty percent of the final peptide suspension was injected onto a 5-cm PepMap trap column and then a Thermo Easy-Spray $75 \mu \mathrm{m} \times 25 \mathrm{~cm} \mathrm{C}-18$ column and separated on a 110 -min gradient (3-28\% ACN), B was increased to $40 \%$ over 2 min, up to $90 \%$ over $2 \mathrm{~min}$, held at $90 \%$ for $2 \mathrm{~min}$, and decreased back to $3 \%$ using an Ultimate 3000 . The Thermo Q Exactive Plus MS ESI parameters were as follows: $350-1700 \mathrm{~m} / \mathrm{z}$, 3e6 AGC MS1, 100-ms MS1 max inject time, 1e5 AGC MS2, 17,500 resolutions, 75-ms MS2 max inject time, 15 loop count, $1.8-\mathrm{m} / \mathrm{z}$ isolation window, NCE $27 \%, 30-\mathrm{s}$ dynamic exclusions, excluding unassigned, 1,8 , and $>8$ charge states. Thermo MS raw files were processed for label-free quantification by MaxQuant LFQ (MaxQuant version 1.6.10.43) with default parameters, using a Uniprot/Swiss-Prot human database, fixed carbidomethyl (C) and variable oxidation (M), and Acetyl (Protein N-term) modifications. Matching between runs was enabled with a match time window of $3 \mathrm{~min}$. Kinase LFQ intensities with two or more unique + razor peptides were processed using Perseus (version 1.6.10.50) - LFQ intensities were $\log _{2}$-transformed and if three values were present in at least one group (siControl or siSOX4), missing values were imputed by column using default parameters. Unpaired Student's $t$ test was performed in Perseus to report $\log _{2}$ fold change and the associated $p$ value.

\section{Next-generation RNA sequencing and data analysis}

Total RNA was extracted from HCC1143 cells treated with either siRNA targeting SOX4 or non-targeting control siRNA for $96 \mathrm{~h}$. RNA libraries were prepared using the NuGen Ovation Universal RNA-Seq System (Catalog \# $0343-32)$ and paired-end ( $2 \times 48 \mathrm{bp}$ ) sequencing was performed using the Illumina NextSeq system. Paired-end FASTQ files were processed using default parameters of Kallisto version v0.43.1, with 100 bootstraps for the expectation-maximization algorithm. Kallisto transcriptome index was built using the Ensembl human genome reference build 38. Gene level summarization of Kallisto abundance (TPM) was established using the $R$ package Tximport v1.0.3 and R v3.3.1.

\section{Chromatin immunoprecipitation (ChIP)}

ChIP was performed as described previously ${ }^{72}$. Briefly, cells were crosslinked with formaldehyde for $10 \mathrm{~min}$ at room temperature. Crosslinking was quenched by $2.5-\mathrm{M}$ glycine and incubated for $5 \mathrm{~min}$ at room temperature. After formaldehyde crosslinking, cells were washed with PBS and nuclei isolated in a buffer containing 50-mM HEPES-KOH, $\mathrm{pH} 7.5,140-$ $\mathrm{mM} \mathrm{NaCl}, 1-\mathrm{mM}$ EDTA, $10 \%$ glycerol, 0.5\% NP40, 0.25\% TritonX, and protease inhibitors. Nuclei were washed with a buffer containing $10-\mathrm{mM}$ Tris- $\mathrm{HCl}, \mathrm{pH}$ 8.0, 200-mM NaCl, 1-mM EDTA, 0.5-mM EGTA, and protease inhibitors. Lysis and immunoprecipitations were carried out in a buffer containing 10-mM Tris- $\mathrm{HCl}, \mathrm{pH}$ 8.0, $100-\mathrm{mM} \mathrm{NaCl}$, 9-mM EDTA, 0.5-mM EGTA, $0.1 \%$ Na-Deoxycholate, $0.5 \%$ N-lauryolsarcosine, and $1.1 \%$ Triton $X$ 100. Complexes were washed five times in RIPA buffer (50-mM HEPES, 500mM LiCl, 0.1-mM EDTA, 1.0\% NP40, and 0.7\% Na-Deoxycholate) and once with TE buffer containing 50-mM NaCl. To elute, beads were incubated with agitation at $65^{\circ} \mathrm{C}$ for $30 \mathrm{~min}$. Two hundred microliter eluate was removed to a fresh tube, and all samples were reverse-crosslinked overnight at $65^{\circ} \mathrm{C}$ for a minimum of $12 \mathrm{~h}$, but not more than $18 \mathrm{~h}$. Two hundred microliter $1 \times$ TE was added to reverse-crosslinked DNA to dilute SDS, and samples were RNaseA-treated (final $0.2-\mathrm{mg} / \mathrm{mL}$ RNase; $37^{\circ} \mathrm{C}$ for $2 \mathrm{~h}$ ) and Proteinase $\mathrm{K}$ (final $0.2-\mathrm{mg} / \mathrm{mL}$ Proteinase $\mathrm{K} ; 55^{\circ} \mathrm{C}$ for $2 \mathrm{~h}$ ) before phenol:chloroform extraction and resuspension in 10-mM Tris- $\mathrm{HCl} \mathrm{pH} \mathrm{8.0.}$ For ChIP-Western blot, the samples were reverse-crosslinked overnight at $65^{\circ} \mathrm{C}$ and about $35-50 \mu \mathrm{g}$ of protein sample was loaded on 4-20\% Miniprotean TGX gradient gel (BioRad). The enrichment of SOX4 and SMARCA4 on TGFBR2 promoter and enhancer was calculated by ChIP-qPCR using gene-specific primers, normalized to the negative control region and presented relative to either non-specific lgG control or no dox treatment. The PNOC promoter was selected as negative control region as it has been previously noted to be predominantly expressed in tissue from the central nervous system but is not expressed in human breast tissues ${ }^{73}$. Primers used are human TGFBR2 promoter-Forward: $5^{\prime}$ - TATCATGGCAAAGACCA CCA-3', Reverse: 5'- CCTACATTTGCCCAAGTTCC-3'; human TGFBR2 enhancer-Forward: 5'- GGGCTATGGCTCTACTGGAA-3', Reverse: 5'- AAGGCTGTA CCCCTGGAAAG-3' and human PNOC promoter-Forward: $5^{\prime}$ - GCTTGAGCT CCTTGGATGAC -3', Reverse: 5'- CCTGTCCCTTACTGCAGA-3'.

\section{Analyses of ChIP-seq data}

SMARCA4 (GSE72141), H3K27ac and H3K4me3 (GSE85158) ChIP-Seq raw data from MDA-MB-231 cells ${ }^{51,52}$ were acquired from GEO. The data were reanalyzed and the FASTQ files were aligned using BowTie2 (v2.2.6) ${ }^{74}$ to UCSC hg19 genome. Significantly enriched peaks were called using MACS (v1.4.2 20120305) $)^{75}$. Genes associated with significantly enriched peaks were determined using GREAT (v3.0.0) to identify genes within $10 \mathrm{~kb}$ of the summit centered peaks ${ }^{76}$ and visualized using $\mathrm{IGV}^{77}$.

\section{Chromatin accessibility assay}

Chromatin accessibility assays were performed as described previously ${ }^{48,78}$ with minor modifications. Approximately $1.0 \times 10^{6}$ HCC1143 or HCC1954 cells were subcultured for siRNA-mediated knockdown of SOX4 for $96 \mathrm{~h}$. Following knockdown, nuclei were collected in nucleus preparation buffer (300-mM sucrose, $10-\mathrm{mM}$ Tris- $\mathrm{HCl}$ at $\mathrm{pH} 7.5,15-\mathrm{mM} \mathrm{NaCl}, 60-\mathrm{mM} \mathrm{KCl}, 5-$ $\mathrm{mM} \mathrm{MgCl}$, 0.1-mM EDTA, 0.15-mM spermine, 0.5-mM spermidine, $0.1 \%$ Nonidet-P40, 0.5- $\mu \mathrm{M}$ phenylmethyl sulfonyl fluoride) supplemented with 3$\mathrm{mM} \mathrm{CaCl} 2$, pelleted by centrifugation, resuspended in MNase digestion buffer (nucleus preparation buffer without Nonidet-P40 and supplemented with $3-\mathrm{mM} \mathrm{CaCl} 2) \pm 5 \mathrm{U}$ of MNase (Sigma, N3755), and incubated for $10 \mathrm{~min}$ at $37^{\circ} \mathrm{C}$. Reactions were terminated with 20- $\mu$ l stop solution (100mM EDTA, 10-mM EGTA [pH 8.1]) and 10- $\mu$ SDS 10\% (w/v). Chromatin was incubated for $5 \mathrm{~h}$ at $65{ }^{\circ} \mathrm{C}$ with proteinase $\mathrm{K}$ to reverse cross-link and remove protein followed by incubation with RNaseA to remove RNA. DNA was extracted with phenol-chloroform-isoamyl alcohol extraction followed by isopropanol precipitation and resuspended in 10-mM Tris $[\mathrm{pH}$ 8.0]. DNA recovered from "Cut" (+MNase) and "Uncut" (-MNase) samples were used in qRT-PCR assays to measure the relative abundance of targeted regions using gene-specific primer pairs. Chromatin accessibility ratios were calculated by determining the "Uncut" to "Cut" ratio for independent pairs of samples, normalized to the negative control region, PNOC promoter, and presented relative to either non-specific IgG control or no dox treatment.

\section{Statistical analysis}

Data are represented as mean and standard deviation of at least three independent experiments. Statistical analyses were carried out with Graphpad Prism version 7.0 and differences were analyzed by unpaired two-tailed $t$-test between two groups and by two-way ANOVA followed by Tukey pairwise comparison test for differences between more than two groups.

\section{DATA AVAILABILITY}

The data generated and analysed during this study are described in the following data record: https://doi.org/10.6084/m9.figshare.14141474 ${ }^{79}$. RNAseq data have been deposited in the Gene Expression Omnibus (GEO) under accession number https:// identifiers.org/geo:GSE158295 ${ }^{80}$. Proteomic data from MIB/MS (https://identifiers.org/ pride.project:PXD022596 ${ }^{81}$ ) and IP-MS (https://identifiers.org/pride.project: 
PXD022811 ${ }^{82}$ ) analyses have been made available through the PRIDE database. SMARCA4 (https://identifiers.org/geo:GSE72141 ${ }^{83}$ ), H3K27ac and H3K4me3 (https:// identifiers.org/geo:GSE85158 84 ) ChIP-Seq raw data from MDA-MB-231 cells were acquired from Gene Expression Omnibus (GEO). The data for Figs. 1, 2, and 5 are presented in Supplementary Data 1-5, which are also available in Excel format as part of the figshare data record.

Received: 21 May 2020; Accepted: 15 March 2021; Published online: 09 April 2021

\section{REFERENCES}

1. Foulkes, W. D., Smith, I. E. \& Reis-Filho, J. S. Triple-negative breast cancer. N. Engl. J. Med. 363, 1938-1948 (2010).

2. Carey, L. A. et al. Race, breast cancer subtypes, and survival in the Carolina Breast Cancer Study. JAMA 295, 2492-2502 (2006).

3. Cancer Genome Atlas, N. Comprehensive molecular portraits of human breast tumours. Nature 490, 61-70 (2012).

4. Lopez-Knowles, E. et al. PI3K pathway activation in breast cancer is associated with the basal-like phenotype and cancer-specific mortality. Int J. Cancer 126, 1121-1131 (2010).

5. Mehta, G. A. et al. Amplification of SOX4 promotes PI3K/Akt signaling in human breast cancer. Breast Cancer Res. Treat. 162, 439-450 (2017).

6. Zhang, Y. et al. A pan-cancer proteogenomic Atlas of PI3K/AKT/mTOR pathway alterations. Cancer Cell 31, 820-832.e3 (2017).

7. Fruman, D. A. \& Rommel, C. PI3K and cancer: lessons, challenges and opportunities. Nat. Rev. Drug Discov. 13, 140-156 (2014).

8. Hallstrom, T. C., Mori, S. \& Nevins, J. R. An E2F1-dependent gene expression program that determines the balance between proliferation and cell death. Cancer Cell 13, 11-22 (2008).

9. Samuels, Y. \& Ericson, K. Oncogenic PI3K and its role in cancer. Curr. Opin. Oncol. 18, 77-82 (2006).

10. Baselga, J. et al. Everolimus in postmenopausal hormone-receptor-positive advanced breast cancer. N. Engl. J. Med. 366, 520-529 (2012).

11. Piccart, M. et al. Everolimus plus exemestane for hormone-receptor-positive, human epidermal growth factor receptor-2-negative advanced breast cancer: overall survival results from BOLERO-2dagger. Ann. Oncol. 25, 2357-2362 (2014).

12. Rodon, J., Dienstmann, R., Serra, V. \& Tabernero, J. Development of PI3K inhibitors: lessons learned from early clinical trials. Nat. Rev. Clin. Oncol. 10, 143-153 (2013).

13. Serra, V. et al. NVP-BEZ235, a dual PI3K/mTOR inhibitor, prevents $\mathrm{PI} 3 \mathrm{~K}$ signaling and inhibits the growth of cancer cells with activating PI3K mutations. Cancer Res. 68, 8022-8030 (2008).

14. Wong, K. K., Engelman, J. A. \& Cantley, L. C. Targeting the PI3K signaling pathway in cancer. Curr. Opin. Genet Dev. 20, 87-90 (2010).

15. Zardavas, D., Fumagalli, D. \& Loi, S. Phosphatidylinositol 3-kinase/AKT/mammalian target of rapamycin pathway inhibition: a breakthrough in the management of luminal (ER+/HER2-) breast cancers? Curr. Opin. Oncol. 24, 623-634 (2012).

16. Mehta, G. A., Khanna, P. \& Gatza, M. L. Emerging role of SOX proteins in breast cancer development and maintenance. J. Mammary Gland Biol. Neoplasia 24, 213-230 (2019)

17. Tiwari, N. et al. Sox4 is a master regulator of epithelial-mesenchymal transition by controlling Ezh2 expression and epigenetic reprogramming. Cancer Cell 23, 768-783 (2013).

18. Vervoort, S. J. et al. Global transcriptional analysis identifies a novel role for SOX4 in tumor-induced angiogenesis. Elife 7, https://doi.org/10.7554/eLife.27706 (2018).

19. Vervoort, S. J. et al. SOX4 can redirect TGF-beta-mediated SMAD3-transcriptional output in a context-dependent manner to promote tumorigenesis. Nucleic Acids Res. 46, 9578-9590 (2018).

20. Zhang, J. et al. SOX4 induces epithelial-mesenchymal transition and contributes to breast cancer progression. Cancer Res. 72, 4597-4608 (2012).

21. Bilir, B. et al. SOX4 is essential for prostate tumorigenesis initiated by PTEN ablation. Cancer Res. 76, 1112-1121 (2016).

22. Scharer, C. D. et al. Genome-wide promoter analysis of the SOX4 transcriptional network in prostate cancer cells. Cancer Res. 69, 709-717 (2009).

23. Ramezani-Rad, P. et al. SOX4 enables oncogenic survival signals in acute lymphoblastic leukemia. Blood 121, 148-155 (2013).

24. Dai, W. et al. SOX4 promotes proliferative signals by regulating glycolysis through AKT activation in melanoma cells. J. Invest. Dermatol. 137, 2407-2416 (2017)

25. Song, G. D., Sun, Y., Shen, H. \& Li, W. SOX4 overexpression is a novel biomarker of malignant status and poor prognosis in breast cancer patients. Tumour Biol. 36, 4167-4173 (2015)
26. Imbalzano, A. N., Kwon, H., Green, M. R. \& Kingston, R. E. Facilitated binding of TATA-binding protein to nucleosomal DNA. Nature 370, 481-485 (1994).

27. Kwon, H., Imbalzano, A. N., Khavari, P. A., Kingston, R. E. \& Green, M. R. Nucleosome disruption and enhancement of activator binding by a human SW1/SNF complex. Nature 370, 477-481 (1994).

28. Kadoch, C. \& Crabtree, G. R. Mammalian SWI/SNF chromatin remodeling complexes and cancer: Mechanistic insights gained from human genomics. Sci. Adv. 1, e1500447 (2015).

29. Kadoch, C. et al. Proteomic and bioinformatic analysis of mammalian SWI/SNF complexes identifies extensive roles in human malignancy. Nat. Genet. 45, 592-601 (2013).

30. Wilson, B. G. \& Roberts, C. W. SWI/SNF nucleosome remodellers and cancer. Nat. Rev. Cancer 11, 481-492 (2011).

31. Bai, J. et al. BRG1 is a prognostic marker and potential therapeutic target in human breast cancer. PLOS ONE 8, e59772 (2013).

32. Do, S. I. et al. Increased Brahma-related Gene 1 expression predicts distant metastasis and shorter survival in patients with invasive ductal carcinoma of the breast. Anticancer Res. 36, 4873-4882 (2016).

33. Guerrero-Martinez, J. A. \& Reyes, J. C. High expression of SMARCA4 or SMARCA2 is frequently associated with an opposite prognosis in cancer. Sci. Rep. 8, 2043 (2018).

34. Wu, Q. et al. The BRG1 ATPase of human SWI/SNF chromatin remodeling enzymes as a driver of cancer. Epigenomics 9, 919-931 (2017).

35. Wu, Q. et al. The BRG1 chromatin remodeling enzyme links cancer cell metabolism and proliferation. Oncotarget 7, 38270-38281 (2016).

36. Curtis, C. et al. The genomic and transcriptomic architecture of 2,000 breast tumours reveals novel subgroups. Nature 486, 346-352 (2012).

37. Parker, J. S. et al. Supervised risk predictor of breast cancer based on intrinsic subtypes. J. Clin. Oncol. 27, 1160-1167 (2009).

38. Gatza, M. L. et al. A pathway-based classification of human breast cancer. Proc. Natl Acad. Sci. USA. 107, 6994-6999 (2010).

39. Gatza, M. L., Silva, G. O., Parker, J. S., Fan, C. \& Perou, C. M. An integrated genomics approach identifies drivers of proliferation in luminal-subtype human breast cancer. Nat. Genet. 46, 1051-1059 (2014).

40. Hoeflich, K. P. et al. In vivo antitumor activity of MEK and phosphatidylinositol 3kinase inhibitors in basal-like breast cancer models. Clin. Cancer Res. 15, 4649-4664 (2009).

41. Duncan, J. S. et al. Dynamic reprogramming of the kinome in response to targeted MEK inhibition in triple-negative breast cancer. Cell 149, 307-321 (2012).

42. Ghosh-Choudhury, N. et al. Requirement of BMP-2-induced phosphatidylinositol 3-kinase and Akt serine/threonine kinase in osteoblast differentiation and Smaddependent BMP-2 gene transcription. J. Biol. Chem. 277, 33361-33368 (2002).

43. von Willebrand, $M$. et al. Modification of phosphatidylinositol 3-kinase SH2 domain binding properties by Abl- or Lck-mediated tyrosine phosphorylation at Tyr-688. J. Biol. Chem. 273, 3994-4000 (1998).

44. Bauer, J. et al. Activin and TGFbeta use diverging mitogenic signaling in advanced colon cancer. Mol. Cancer 14, 182 (2015).

45. Zecchin, D. et al. Combined targeting of G protein-coupled receptor and EGF receptor signaling overcomes resistance to PI3K pathway inhibitors in PTEN-null triple negative breast cancer. EMBO Mol. Med. 12, e11987 (2020).

46. Cutter, A. R. \& Hayes, J. J. A brief review of nucleosome structure. FEBS Lett. 589, 2914-2922 (2015).

47. Roberts, C. W. \& Orkin, S. H. The SWI/SNF complex-chromatin and cancer. Nat. Rev. Cancer 4, 133-142 (2004).

48. Pfister, N. T. et al. Mutant p53 cooperates with the SWI/SNF chromatin remodeling complex to regulate VEGFR2 in breast cancer cells. Genes Dev. 29, 1298-1315 (2015).

49. Sokol, E. S. et al. SMARCE1 is required for the invasive progression of in situ cancers. Proc. Natl Acad. Sci. USA. 114, 4153-4158 (2017).

50. Wang, L. et al. CARM1 methylates chromatin remodeling factor BAF155 to enhance tumor progression and metastasis. Cancer Cell 25, 21-36 (2014).

51. Takaku, M. et al. GATA3-dependent cellular reprogramming requires activationdomain dependent recruitment of a chromatin remodeler. Genome Biol. 17, 36 (2016).

52. Franco, H. L. et al. Enhancer transcription reveals subtype-specific gene expression programs controlling breast cancer pathogenesis. Genome Res. 28, 159-170 (2018).

53. Marathe, H. G. et al. BRG1 interacts with SOX10 to establish the melanocyte lineage and to promote differentiation. Nucleic Acids Res. 45, 6442-6458 (2017).

54. Nie, Z. et al. A specificity and targeting subunit of a human SWI/SNF familyrelated chromatin-remodeling complex. Mol. Cell Biol. 20, 8879-8888 (2000).

55. Smith, D. K., Yang, J., Liu, M. L. \& Zhang, C. L. Small molecules modulate chromatin accessibility to promote NEUROG2-mediated fibroblast-to-neuron reprogramming. Stem Cell Rep. 7, 955-969 (2016)

56. Vervoort, S. J., van Boxtel, R. \& Coffer, P. J. The role of SRY-related HMG box transcription factor 4 (SOX4) in tumorigenesis and metastasis: friend or foe? Oncogene 32, 3397-3409 (2013). 
57. Wu, Q. et al. The SWI/SNF ATPases are required for triple negative breast cancer cell proliferation. J. Cell Physiol. 230, 2683-2694 (2015).

58. Huang, F. \& Chen, Y. G. Regulation of TGF-beta receptor activity. Cell Biosci. 2, 9 (2012).

59. Bhola, N. E. et al. TGF-beta inhibition enhances chemotherapy action against triple-negative breast cancer. J. Clin. Invest 123, 1348-1358 (2013).

60. Bierie, B. et al. Transforming growth factor-beta regulates mammary carcinoma cell survival and interaction with the adjacent microenvironment. Cancer Res. 68, 1809-1819 (2008).

61. Derynck, R. \& Zhang, Y. E. Smad-dependent and Smad-independent pathways in TGF-beta family signalling. Nature 425, 577-584 (2003).

62. Yi, J. Y., Shin, I. \& Arteaga, C. L. Type I transforming growth factor beta receptor binds to and activates phosphatidylinositol 3-kinase. J. Biol. Chem. 280, 10870-10876 (2005).

63. Huang, S. et al. MED12 controls the response to multiple cancer drugs through regulation of TGF-beta receptor signaling. Cell 151, 937-950 (2012)

64. Hachim, I. Y. et al. Transforming growth factor-beta regulation of ephrin type-A receptor 4 signaling in breast cancer cellular migration. Sci. Rep. 7, 14976 (2017)

65. Carracedo, A. et al. Inhibition of mTORC1 leads to MAPK pathway activation through a PI3K-dependent feedback loop in human cancer. J. Clin. Invest. 118, 3065-3074 (2008).

66. Tolcher, A. W. et al. Antitumor activity in RAS-driven tumors by blocking AKT and MEK. Clin. Cancer Res. 21, 739-748 (2015).

67. Saini, K. S. et al. Targeting the PI3K/AKT/mTOR and Raf/MEK/ERK pathways in the treatment of breast cancer. Cancer Treat. Rev. 39, 935-946 (2013).

68. Hoadley, K. A. et al. Multiplatform analysis of 12 cancer types reveals molecular classification within and across tissues of origin. Cell 158, 929-944 (2014).

69. Reich, M. et al. GenePattern 2.0. Nat. Genet. 38, 500-501 (2006).

70. Nusinow, D. P. et al. Quantitative proteomics of the cancer cell line encyclopedia. Cell 180, 387-402.e16 (2020).

71. Mehta, G. et al. MITF interacts with the SWI/SNF subunit, BRG1, to promote GATA4 expression in cardiac hypertrophy. J. Mol. Cell Cardiol. 88, 101-110 (2015).

72. Lee, T. I., Johnstone, S. E. \& Young, R. A. Chromatin immunoprecipitation and microarray-based analysis of protein location. Nat. Protoc. 1, 729-748 (2006).

73. Mollereau, C. et al. Structure, tissue distribution, and chromosomal localization of the prepronociceptin gene. Proc. Natl Acad. Sci. USA. 93, 8666-8670 (1996).

74. Langmead, B. \& Salzberg, S. L. Fast gapped-read alignment with Bowtie 2. Nat. Methods 9, 357-359 (2012).

75. Zhang, Y. et al. Model-based analysis of ChIP-Seq (MACS). Genome Biol. 9, R137 (2008).

76. McLean, C. Y. et al. GREAT improves functional interpretation of cis-regulatory regions. Nat. Biotechnol. 28, 495-501 (2010).

77. Thorvaldsdottir, H., Robinson, J. T. \& Mesirov, J. P. Integrative Genomics Viewer (IGV): high-performance genomics data visualization and exploration. Brief Bioinform. 14, 178-192 (2013).

78. Cruickshank, M., Fenwick, E., Abraham, L. J. \& Ulgiati, D. Quantitative differences in chromatin accessibility across regulatory regions can be directly compared in distinct cell-types. Biochem. Biophys. Res. Commun. 367, 349-355 (2008).

79. Mehta, G. A. et al. Metadata record for the manuscript: SOX4 and SMARCA4 cooperatively regulate $\mathrm{PI} 3 \mathrm{~K}$ signaling through transcriptional activation of TGFBR2. https://doi.org/10.6084/m9.figshare.14141474 (2021).

80. Gene Expression Omnibus. GSE158295 (2021).

81. PRIDE. PXD022596 (2021)

82. PRIDE. PXD022811 (2021).

83. Gene Expression Omnibus. GSE72141 (2016).

84. Gene Expression Omnibus. GSE85158 (2017).

\section{ACKNOWLEDGEMENTS}

We would like to thank the members of our laboratory and colleagues for their comments and suggests on this project and for critical review of the manuscript. We also would like to thank Dr Emma Doud for her help with MIB/MS mass spec work This work was supported by R00-CA166228 from the National Cancer Institute (NCI) of the National Institutes of Health (NIH), V2016-013 from the V Foundation for Cancer Research and 133887-RSG-19-160-01-TBE from the American Cancer Society to MLG as well as DHFS-17PPC-002 from the New Jersey Commission for Cancer Research to GAM. Rutgers Cancer Institute Genome Editing Shared Resource is supported by Cancer Center Support Grant P30-CA072720-5922 from the NCl. The Center for Advanced Proteomics Research at Rutgers Newark is funded, in part, by NS046593 and 1S10OD025047-01 (NIH). The Indiana University Proteomics Core is supported by the Indiana Clinical and Translational Sciences Institute funded, in part by UL1TR002529 from the NIH National Center for Advancing Translational Sciences, Clinical and Translational Sciences Award and the Cancer Center Support Grant for the IU Simon Comprehensive Cancer Center (P30-CA082709) from NCI.

\section{AUTHOR CONTRIBUTIONS}

GAM and MLG conceived and designed the study. GAM and MLG performed computational analyses. GAM, SAD, SPA, and GLJ were responsible for the MIB/MS studies. GAM, CAK, and MLG were responsible for the RNAseq experimental design, execution, and analyses. GAM, KT, and MPV were responsible for the analysis of the ChIP-seq data. GAM and PK were responsible for experimental analysis. MLG supervised all research. GAM and MLG wrote the manuscript. All authors have reviewed and approved the final manuscript.

\section{COMPETING INTERESTS}

The authors declare no competing interests.

\section{ADDITIONAL INFORMATION}

Supplementary information The online version contains supplementary materia available at https://doi.org/10.1038/s41523-021-00248-2.

Correspondence and requests for materials should be addressed to M.L.G.

Reprints and permission information is available at http://www.nature.com/ reprints

Publisher's note Springer Nature remains neutral with regard to jurisdictional claims in published maps and institutional affiliations.

Open Access This article is licensed under a Creative Commons Attribution 4.0 International License, which permits use, sharing, adaptation, distribution and reproduction in any medium or format, as long as you give appropriate credit to the original author(s) and the source, provide a link to the Creative Commons license, and indicate if changes were made. The images or other third party material in this article are included in the article's Creative Commons license, unless indicated otherwise in a credit line to the material. If material is not included in the article's Creative Commons license and your intended use is not permitted by statutory regulation or exceeds the permitted use, you will need to obtain permission directly from the copyright holder. To view a copy of this license, visit http://creativecommons. org/licenses/by/4.0/.

(c) The Author(s) 2021 